\title{
NOTES ON THE WEAK POSITIVITY THEOREMS
}

\author{
OSAMU FUJINO
}

\begin{abstract}
We discuss the (twisted) weak positivity theorem. We also treat some applications.
\end{abstract}

\section{Contents}

1. Introduction 1

2. Preliminaries 6

3. Fundamental injectivity theorem 11

4. MHS for cohomology with compact support 13

5. Injectivity, torsion-free, and vanishing theorems 18

6. Semipositivity theorem 19

7. Weakly positive sheaves 20

8. Twisted weak positivity 27

9. Addition formula 34

10. Addition for logarithmic Kodaira dimensions 36

References $\quad 40$

\section{INTRODUCTION}

In this paper, we discuss the (twisted) weak positivity theorem. We give a detailed proof of the following theorem, which is essentially equivalent to [Ca, Theorem 4.13] (see also [L]). The proof is based on our semipositivity theorem (see Theorem 1.5, [F1], [FF], and [FFS]). Note that Theorem 1.1 has already played important roles in [HM], [FG], and so on, when $X$ is projective (see also [KP]).

Theorem 1.1 (Twisted weak positivity). Let $(X, \Delta)$ be a log canonical pair such that $X$ is in Fujiki's class $\mathcal{C}$ and let $f: X \rightarrow Y$ be a surjective

Date: $2015 / 6 / 30$, version 0.54 .

2010 Mathematics Subject Classification. Primary 14J10; Secondary 14D07, $32 \mathrm{G} 20$.

Key words and phrases. semipositivity theorem, weak positivity, mixed Hodge structures, Iitaka's conjecture. 
morphism onto a smooth projective variety $Y$. Assume that $k\left(K_{X}+\Delta\right)$ is Cartier. Then, for every positive integer $m$,

$$
f_{*} \mathcal{O}_{X}\left(m k\left(K_{X / Y}+\Delta\right)\right)
$$

is weakly positive.

We have already discussed some generalizations of Theorem 1.1 in [F9], where $Y$ is a curve and $X$ is a reducible variety. They play crucial roles in order to prove the projectivity of various moduli spaces. For the details, see [F9] and [KP]. For the basic properties of weakly positive sheaves and Viehweg's fundamental results, we recommend the reader to see $[\mathrm{F} 15]$.

In this paper, we first prove the following Hodge theoretic injectivity theorem (cf. [EV], [A], [F10], etc.).

Theorem 1.2 (Fundamental injectivity theorem). Let $X$ be a complex manifold in Fujiki's class $\mathcal{C}$ and let $\Delta$ be a boundary $\mathbb{R}$-divisor on $X$ such that $\operatorname{Supp} \Delta$ is a simple normal crossing divisor on $X$. Let $\mathcal{L}$ be a line bundle on $X$ and let $D$ be an effective Weil divisor on $X$ whose support is contained in $\operatorname{Supp} \Delta$. Assume that $\mathcal{L} \sim_{\mathbb{R}} K_{X}+\Delta$. Then the natural homomorphism

$$
H^{q}(X, \mathcal{L}) \rightarrow H^{q}\left(X, \mathcal{L} \otimes \mathcal{O}_{X}(D)\right)
$$

induced by the inclusion $\mathcal{O}_{X} \rightarrow \mathcal{O}_{X}(D)$ is injective for every $q$.

It is easy to see that Theorem 1.2 implies:

Theorem 1.3 (Injectivity theorem). Let $X$ be a complex manifold in Fujiki's class $\mathcal{C}$ and let $\Delta$ be a boundary $\mathbb{R}$-divisor such that $\operatorname{Supp} \Delta$ is simple normal crossing. Let $\mathcal{L}$ be a line bundle on $X$ and let $D$ be an effective Cartier divisor whose support contains no log canonical centers of $(X, \Delta)$. Assume the following conditions.

(i) $\mathcal{L} \sim_{\mathbb{R}} K_{X}+\Delta+H$,

(ii) $H$ is a semi-ample $\mathbb{R}$-divisor, and

(iii) $t H \sim_{\mathbb{R}} D+D^{\prime}$ for some positive real number $t$, where $D^{\prime}$ is an effective $\mathbb{R}$-divisor whose support contains no log canonical centers of $(X, \Delta)$.

Then the homomorphisms

$$
H^{q}(X, \mathcal{L}) \rightarrow H^{q}\left(X, \mathcal{L} \otimes \mathcal{O}_{X}(D)\right)
$$

induced by the natural inclusion $\mathcal{O}_{X} \rightarrow \mathcal{O}_{X}(D)$ are injective for all $q$.

As an application of Theorem 1.3, we obtain: 
Theorem 1.4 (Torsion-freeness and vanishing theorem). Let $Y$ be a complex manifold in Fujiki's class $\mathcal{C}$ and let $\Delta$ be a boundary $\mathbb{R}$-divisor such that $\operatorname{Supp} \Delta$ is simple normal crossing. Let $f: Y \rightarrow X$ be a surjective morphism onto a projective variety $X$ and let $\mathcal{L}$ be a line bundle on $Y$ such that $\mathcal{L}-\left(K_{Y}+\Delta\right)$ is f-semi-ample.

(i) Let $q$ be an arbitrary nonnegative integer. Then every associated prime of $R^{q} f_{*} \mathcal{L}$ is the generic point of the $f$-image of some log canonical stratum of $(Y, \Delta)$.

(ii) Assume that $\mathcal{L}-\left(K_{Y}+\Delta\right) \sim_{\mathbb{R}} f^{*} H$ for some ample $\mathbb{R}$-divisor $H$ on $X$. Then $H^{p}\left(X, R^{q} f_{*} \mathcal{L}\right)=0$ for every $p>0$ and $q \geq 0$.

When $X$ and $Y$ are projective, Theorem 1.3 and Theorem 1.4 are well-known and play crucial roles in [F6].

By using Theorem 1.4, we can establish:

Theorem 1.5 (Semipositivity theorem). Let $X$ be a compact Kähler manifold and let $Y$ be a smooth projective variety, and let $f: X \rightarrow Y$ be a surjective morphism. Let $D$ be a simple normal crossing divisor on $X$ such that every stratum of $D$ is dominant onto $Y$. Let $\Sigma$ be a simple normal crossing divisor on $Y$. We put $Y_{0}=Y \backslash \Sigma$. If $f$ is smooth and $D$ is relatively normal crossing over $Y_{0}$, then $R^{i} f_{*} \omega_{X / Y}(D)$ is the upper canonical extension of the bottom Hodge filtration. In particular, it is locally free.

We further assume that all the local monodromies on the local system $R^{d+i} f_{0 *} \mathbb{C}_{X_{0}-D_{0}}$ around $\Sigma$ are unipotent, then $R^{i} f_{*} \omega_{X / Y}(D)$ is nef, where $d=\operatorname{dim} X-\operatorname{dim} Y, X_{0}=f^{-1}\left(Y_{0}\right)$, and $D_{0}=\left.D\right|_{X_{0}}$.

We note that a nef locally free sheaf was originally called a (numerically) semipositive locally free sheaf in the literature. Theorem 1.5 is the main ingredient of Theorem 1.1. In this paper, we do not use [Kw1, Theorem 32] for the proof of Theorem 1.1 (see Remark 6.4). Note that Theorem 7.8 and Corollary 7.11, which directly follow from Theorem 1.5 , are new.

Let us discuss some applications of Theorem 1.1. The following conjecture is a natural formulation of Iitaka's conjecture for the minimal model program.

Conjecture 1.6 (Log Iitaka conjecture). Let $(X, \Delta)$ be a projective log canonical pair and let $f: X \rightarrow Y$ be a surjective morphism onto a normal projective variety $Y$ with connected fibers. Then

$$
\kappa\left(X, K_{X}+\Delta\right) \geq \kappa\left(X_{y}, K_{X_{y}}+\left.\Delta\right|_{X_{y}}\right)+\kappa(Y)
$$


where $X_{y}$ is a sufficiently general fiber of $f: X \rightarrow Y$. Note that $\kappa(Y)$ denotes the Kodaira dimension of $Y$, that is, $\kappa(Y)=\kappa\left(\widetilde{Y}, K_{\widetilde{Y}}\right)$, where $\widetilde{Y} \rightarrow Y$ is a resolution of singularities.

When $\operatorname{dim} X=n$ and $\operatorname{dim} Y=m$ in Conjecture 1.6, we sometimes call it Conjecture $C_{n, m}^{\log }$. If $X$ and $Y$ are smooth and $\Delta=0$, then Conjecture 1.6 is nothing but Iitaka's original conjecture (see [I1]). We can easily check that Conjecture 1.6 holds true when $Y$ is of general type and $\Delta$ is a $\mathbb{Q}$-divisor. Note that Theorem 1.7 below is contained in [Ca] (see also [N2, Chapter V. 4.1. Theorem (2)]). Moreover, Campana raised the orbifold version of the Iitaka conjecture. For the details, see [Ca, Section 4] (see also [L]).

Theorem 1.7 (Addition formula). Let $(X, \Delta)$ be a projective log canonical pair such that $\Delta$ is a $\mathbb{Q}$-divisor and let $f: X \rightarrow Y$ be a surjective morphism onto a normal projective variety $Y$ with connected fibers. Assume that $\kappa(Y)=\operatorname{dim} Y$. Then

$$
\begin{aligned}
\kappa\left(X, K_{X}+\Delta\right) & =\kappa\left(X_{y}, K_{X_{y}}+\left.\Delta\right|_{X_{y}}\right)+\kappa(Y) \\
& =\kappa\left(X_{y}, K_{X_{y}}+\left.\Delta\right|_{X_{y}}\right)+\operatorname{dim} Y
\end{aligned}
$$

where $X_{y}$ is a sufficiently general fiber of $f: X \rightarrow Y$.

Remark 1.8. By Nakayama (see [N2, Chapter V. 4.4. Theorem (1)]), we have

$$
\kappa_{\sigma}\left(X, K_{X}+\Delta\right) \geq \kappa_{\sigma}\left(X_{y}, K_{X_{y}}+\left.\Delta\right|_{X_{y}}\right)+\kappa_{\sigma}\left(\widetilde{Y}, K_{\widetilde{Y}}\right),
$$

where $\kappa_{\sigma}$ denotes Nakayama's numerical dimension. In general, it is conjectured that $\kappa_{\sigma}\left(X, K_{X}+\Delta\right)=\kappa\left(X, K_{X}+\Delta\right)$ when $\Delta$ is a $\mathbb{Q}$ divisor, which is sometimes called the generalized abundance conjecture. If $\kappa_{\sigma}\left(X, K_{X}+\Delta\right)=\kappa\left(X, K_{X}+\Delta\right)$, then we have

$$
\begin{aligned}
\kappa\left(X, K_{X}+\Delta\right) & \geq \kappa_{\sigma}\left(X_{y}, K_{X_{y}}+\left.\Delta\right|_{X_{y}}\right)+\kappa_{\sigma}\left(\widetilde{Y}, K_{\widetilde{Y}}\right) \\
& \geq \kappa\left(X_{y}, K_{X_{y}}+\left.\Delta\right|_{X_{y}}\right)+\kappa\left(\widetilde{Y}, K_{\tilde{Y}}\right) .
\end{aligned}
$$

Therefore, Conjecture 1.6 follows from the generalized abundance conjecture when $\Delta$ is a $\mathbb{Q}$-divisor.

Theorem 1.9 is due to Maehara (see [Ma, Corollary 2]). In this paper, we recover it as an application of Theorem 1.1.

Theorem 1.9 (Addition formula for logarithmic Kodaira dimensions). Let $f: X \rightarrow Y$ be a surjective morphism between smooth projective varieties with connected fibers. Let $D_{X}\left(r e s p . D_{Y}\right)$ be a simple normal 
crossing divisor on $X$ (resp. $Y)$. Assume that $\operatorname{Supp} f^{*} D_{Y} \subset \operatorname{Supp} D_{X}$. We further assume that $\kappa\left(Y, K_{Y}+D_{Y}\right)=\operatorname{dim} Y$. Then we have

$$
\begin{aligned}
\kappa\left(X, K_{X}+D_{X}\right) & =\kappa\left(F, K_{F}+\left.D_{X}\right|_{F}\right)+\kappa\left(Y, K_{Y}+D_{Y}\right) \\
& =\kappa\left(F, K_{F}+\left.D_{X}\right|_{F}\right)+\operatorname{dim} Y,
\end{aligned}
$$

where $F$ is a sufficiently general fiber of $f: X \rightarrow Y$.

We put $X^{0}=X \backslash D_{X}, Y^{0}=Y \backslash D_{Y}$, and $F^{0}=\left.F\right|_{X^{0}}$. Then the above equality is nothing but

$$
\begin{aligned}
\bar{\kappa}\left(X^{0}\right) & =\bar{\kappa}\left(F^{0}\right)+\bar{\kappa}\left(Y^{0}\right) \\
& =\bar{\kappa}\left(F^{0}\right)+\operatorname{dim} Y^{0} .
\end{aligned}
$$

Note that $\bar{\kappa}$ denotes Iitaka's logarithmic Kodaira dimension (see [I2]).

We will quickly prove Theorem 1.7 and Theorem 1.9 in Section 9 and Section 10 respectively by using Theorem 1.1 and [AK]. In [F13], we prove the subadditivity of the logarithmic Kodaira dimension for affine varieties.

We summarize the contents of this paper. Section 2 collects some basic results and definitions. In Section 3, we prove the fundamental injectivity theorem: Theorem 1.2. The proof of Theorem 1.2 uses the theory of mixed Hodge structures. Section 4 is devoted to the theory of mixed Hodge structures for cohomology with compact support. In Section 5, we prove Theorem 1.3 and Theorem 1.4. These are direct consequences of Theorem 1.2. In Section 6, we explain the semipositivity theorem: Theorem 1.5. In Section 7, we discuss weakly positive sheaves. Section 8 is the main part of this paper. It is devoted to the proof of the twisted weak positivity theorem: Theorem 1.1. We prove Theorem 1.7 (resp. Theorem 1.9) in Section 9 (resp. Section 10) as an application of Theorem 1.1.

In this paper, we discuss neither Nakayama's sophisticated treatment of weak positivity in [N2, Chapter V. §3] nor Schnell's results on weak positivity coming from Saito's theory of mixed Hodge modules (see [Schn]). We naively discuss some generalizations of Viehweg's weak positivity following [V2], [Ca], etc. The main motivation of this paper is to understand and clarify Viehweg's clever covering arguments used for the proof of his famous weak positivity theorem (see also [F15]).

Acknowledgments. The author was partially supported by Grantin-Aid for Young Scientists (A) 24684002 from JSPS. He would like to thank Professors Akira Fujiki and Kazuhisa Maehara for answering his questions. He also would like to thank Professor Noboru Nakayama for useful comments and discussions. He thanks the referees for useful 
comments. Finally, he thanks Yoshinori Gongyo for pointing out a typo.

We will use the standard notation of the minimal model program as in [F6]. In this paper, we always assume that complex varieties are Hausdorff and countable at infinity. For the basic theory of complex varieties, see, for example, [BS], [Fi], and [N2]. The style of this paper is the same as that of [F6] (see [F3], [F4], [FF], [F12], [F16], etc.). Our results depend on the theory of variations of mixed Hodge structure (see $[\mathrm{F} 1],[\mathrm{FF}]$, and $[\mathrm{FFS}]$ ).

\section{Preliminaries}

Let us start with some remarks on canonical divisors.

2.1 (Canonical divisors). We consider complex variety $X$, which is not necessarily algebraic.

Remark 2.2. (i) Let $\omega_{X}^{\bullet}$ be the dualizing complex of a complex variety $X$ (see, for example, [RR], [RRV], and [BS]). We put $\omega_{X}=\mathcal{H}^{-d}\left(\omega_{X}^{\bullet}\right)$, where $d=\operatorname{dim} X$, and call it the canonical sheaf of $X$. When $X$ is a compact complex manifold, it is well-known that $\omega_{X} \simeq \Omega_{X}^{d}$. For the details of $\omega_{X}^{\bullet}$, see, for example, [BS, Chapter VII $\left.\S 2\right]$.

(iii) Some complex variety $X$ does not admit any nonzero meromorphic section of $\omega_{X}$. However, if there is no risk of confusion, we use the symbol $K_{X}$ as a formal divisor class with an isomorphism $\mathcal{O}_{X}\left(K_{X}\right) \simeq \omega_{X}$ and call it the canonical divisor of $X$. See [N2, Chapter II. $\$ 4]$.

Remark 2.3. Let $D$ be a Cartier divisor and let $\mathcal{L}$ be a line bundle on a complex variety $X$. If there is no risk of confusion, we sometimes write

$$
\mathcal{O}_{X}\left(K_{X}+D+\mathcal{L}\right)
$$

in order to express

$$
\omega_{X} \otimes \mathcal{O}_{X}(D) \otimes \mathcal{L}
$$

For simplicity, we sometimes use $\mathcal{L}^{N}$ to denote $\mathcal{L}^{\otimes N}$ if there is no risk of confusion.

In this paper, all complex varieties are algebraic or compact. Therefore, there are no subtle problems in the following definitions.

2.4 (Singularities of pairs). Let us recall the definition of singularities of pairs.

Let $X$ be a normal variety and let $\Delta$ be an effective $\mathbb{R}$-divisor on $X$ such that $K_{X}+\Delta$ is $\mathbb{R}$-Cartier. Let $f: Y \rightarrow X$ be a resolution 
such that $\operatorname{Exc}(f) \cup f_{*}^{-1} \Delta$ has a simple normal crossing support, where $\operatorname{Exc}(f)$ is the exceptional locus of $f$ and $f_{*}^{-1} \Delta$ is the strict transform of $\Delta$ on $Y$. We write

$$
K_{Y}=f^{*}\left(K_{X}+\Delta\right)+\sum_{i} a_{i} E_{i}
$$

and $a\left(E_{i}, X, \Delta\right)=a_{i}$. We say that $(X, \Delta)$ is $l c$ if and only if $a_{i} \geq$ -1 for every $i$. Note that the discrepancy $a(E, X, \Delta) \in \mathbb{R}$ can be defined for every prime divisor $E$ over $X$. It is well-known that $(X, \Delta)$ is lc if and only if $a(E, X, \Delta) \geq-1$ for every prime divisor $E$ over $X$. Let $(X, \Delta)$ be an lc pair. If there is a resolution $f: Y \rightarrow X$ such that $\operatorname{Exc}(f)$ is a divisor, $\operatorname{Exc}(f) \cup f_{*}^{-1} \Delta$ has a simple normal crossing support, and $a(E, X, \Delta)>-1$ for every $f$-exceptional divisor $E$, then $(X, \Delta)$ is called $d l t$. Here, lc (resp. dlt) is an abbreviation of log canonical (resp. divisorial log terminal).

For the details and various examples of singularities of pairs, see, for example, [F2] (see also [F12, Section 2.3]).

Remark 2.5 (Szabó's resolution lemma). We note that Szabó's resolution lemma (see, for example, [F2, 3.5 Resolution lemma]) now holds for compact complex varieties. For the details, see, for example, [Ko2, Theorem 10.45, Proposition 10.49, and the proof of (10.45)]. We will use Szabó's resolution lemma repeatedly in this paper.

Let us recall the definition of log canonical centers.

Definition 2.6 (Log canonical center). Let $(X, \Delta)$ be a log canonical pair. If there is a resolution $f: Y \rightarrow X$ and a prime divisor $E$ on $Y$ such that $a(E, X, \Delta)=-1$, then $f(E)$ is called a log canonical center of $(X, \Delta)$.

Definition 2.7 is useful for torsion-free theorem.

Definition 2.7 (Log canonical stratum). Let $(X, \Delta)$ be a log canonical pair. A log canonical stratum (an lc stratum, for short) of $(X, \Delta)$ is $X$ itself or a $\log$ canonical center of $(X, \Delta)$. Note that $X$ is a log canonical stratum of $(X, \Delta)$ but is not a log canonical center of $(X, \Delta)$.

2.8 (Divisors). Let us recall some basic operations for $\mathbb{Q}$-divisors and $\mathbb{R}$-divisors.

For an $\mathbb{R}$-divisor $D=\sum_{i=1}^{r} d_{i} D_{i}$ such that $D_{i}$ is a prime divisor for every $i$ and $D_{i} \neq D_{j}$ for $i \neq j$, we define the round-down $\lfloor D\rfloor=$ $\sum_{i=1}^{r}\left\lfloor d_{i}\right\rfloor D_{i}$ (resp. the round-up $\lceil D\rceil=\sum_{i=1}^{r}\left\lceil d_{i}\right\rceil D_{i}$ ), where for every real number $x,\lfloor x\rfloor$ (resp. $\lceil x\rceil$ ) is the integer defined by $x-1<\lfloor x\rfloor \leq x$ (resp. $x \leq\lceil x\rceil<x+1$ ). The fractional part $\{D\}$ of $D$ denotes $D-\lfloor D\rfloor$. 
We also define $D^{=1}=\sum_{d_{i}=1} D_{i}$. We call $D$ a boundary $\mathbb{R}$-divisor if $0 \leq d_{i} \leq 1$ for every $i$.

Remark 2.9. Let $X$ be a compact complex manifold and let $D_{1}, D_{2}$, $\cdots, D_{k}$ be Cartier divisors on $X$. We consider the linear map

$$
\varphi: \mathbb{R}^{k} \longrightarrow \operatorname{Pic}(X) \otimes \mathbb{R}
$$

defined by $\varphi\left(r_{1}, r_{2}, \cdots, r_{k}\right)=r_{1} D_{1}+r_{2} D_{2}+\cdots+r_{k} D_{k}$, which is defined over $\mathbb{Q}$. Let $\mathcal{L}$ be a line bundle on $X$. Then $\mathcal{L} \sim_{\mathbb{R}} \sum_{i=1}^{k} r_{i} D_{i}$ means $\mathcal{L}=$ $\varphi\left(r_{1}, r_{2}, \cdots, r_{k}\right)$ in $\operatorname{Pic}(X) \otimes \mathbb{R}$. Note that $\varphi^{-1}(\mathcal{L})$ is an affine subspace of $\mathbb{R}^{k}$ defined over $\mathbb{Q}$. Therefore, we can find $\left(r_{1}^{\prime}, r_{2}^{\prime}, \cdots, r_{k}^{\prime}\right) \in \mathbb{Q}^{k}$ such that $\mathcal{L} \sim_{\mathbb{Q}} \sum_{i=1}^{k} r_{i}^{\prime} D_{i}$, that is, $\mathcal{L}=\varphi\left(r_{1}^{\prime}, r_{2}^{\prime}, \cdots, r_{k}^{\prime}\right)$ in $\operatorname{Pic}(X) \otimes \mathbb{Q}$ if $\varphi^{-1}(\mathcal{L})$ is not empty.

2.10 (Fujiki's class $\mathcal{C}$ ). In this paper, we use the notion of complex varieties in Fujiki's class $\mathcal{C}$.

Definition 2.11 (Fujiki's class $\mathcal{C}$ ). Let $X$ be a compact reduced complex analytic space. Then $X$ is in Fujiki's class $\mathcal{C}$ if and only if there is a surjective morphism $f: Y \rightarrow X$ with $Y$ a compact Kähler manifold. It is well-known that $X$ is in Fujiki's class $\mathcal{C}$ if and only if there is a bimeromorphic morphism $g: V \rightarrow X$ from a compact Kähler manifold $V$ (see, for example, [Va, Théorème 3]).

It is well-known that some basic results on the minimal model program can be generalized for varieties in Fujiki's class $\mathcal{C}$. See [N1], [F5, Section 4], etc.

Remark 2.12. For the details of complex varieties in Fujiki's class $\mathcal{C}$, (locally) Kähler morphisms, and so on, see [Fk1], [Fk2], and [Va]. Note that every (locally) projective morphism is (locally) Kähler and that the composition of two locally Kähler morphisms is again locally Kähler (see [Fk2, (1.2), (2.1), (2.2), and so on]).

2.13 (Simple normal crossing varieties). In Section 4, we will use the Mayer-Vietoris simplicial resolution of a simple normal crossing variety $X$ in order to discuss various mixed Hodge structures.

Definition 2.14 (Mayer-Vietoris simplicial resolution). Let $X$ be a simple normal crossing variety with the irreducible decomposition $X=$ $\bigcup_{i \in I} X_{i}$. Let $I_{n}$ be the set of strictly increasing sequences $\left(i_{0}, \cdots, i_{n}\right)$ in $I$ and $X^{n}=\coprod_{I_{n}} X_{i_{0}} \cap \cdots \cap X_{i_{n}}$ the disjoint union of the intersections of $X_{i}$. Let $\varepsilon_{n}: X^{n} \rightarrow X$ be the disjoint union of the natural inclusions. Then $\left\{X^{n}, \varepsilon_{n}\right\}_{n}$ has a natural semi-simplicial structure. The face operator is induced by $\lambda_{j, n}$, where

$$
\lambda_{j, n}: X_{i_{0}} \cap \cdots \cap X_{i_{n}} \rightarrow X_{i_{0}} \cap \cdots \cap X_{i_{j-1}} \cap X_{i_{j+1}} \cap \cdots \cap X_{i_{n}}
$$


is the natural closed embedding for $j \leq n$ (cf. [E2, 3.5.5]). We denote it by $\varepsilon: X^{\bullet} \rightarrow X$ and call it the Mayer-Vietoris simplicial resolution of $X$. The complex

$$
0 \rightarrow \varepsilon_{0 *} \mathcal{O}_{X^{0}} \rightarrow \varepsilon_{1 *} \mathcal{O}_{X^{1}} \rightarrow \cdots \rightarrow \varepsilon_{k *} \mathcal{O}_{X^{k}} \rightarrow \cdots,
$$

where the differential $d_{k}: \varepsilon_{k *} \mathcal{O}_{X^{k}} \rightarrow \varepsilon_{k+1 *} \mathcal{O}_{X^{k+1}}$ is $\sum_{j=0}^{k+1}(-1)^{j} \lambda_{j, k+1}^{*}$ for every $k \geq 0$, is denoted by $\mathcal{O}_{X} \bullet$. We see that $\mathcal{O}_{X} \bullet$ is quasi-isomorphic to $\mathcal{O}_{X}$. By tensoring $\mathcal{L}$, any line bundle on $X$, to $\mathcal{O}_{X} \bullet$, we obtain a complex

$$
0 \rightarrow \varepsilon_{0 *} \mathcal{L}^{0} \rightarrow \varepsilon_{1 *} \mathcal{L}^{1} \rightarrow \cdots \rightarrow \varepsilon_{k *} \mathcal{L}^{k} \rightarrow \cdots,
$$

where $\mathcal{L}^{n}=\varepsilon_{n}^{*} \mathcal{L}$. Here, $\mathcal{L}^{n}$ does not mean $\mathcal{L}^{\otimes n}$ (see Remark 2.3). It is denoted by $\mathcal{L}^{\bullet}$. Of course, $\mathcal{L}^{\bullet}$ is quasi-isomorphic to $\mathcal{L}$. We note that $\mathbb{H}^{q}\left(X, \mathcal{L}^{\bullet}\right)$ is obviously isomorphic to $H^{q}(X, \mathcal{L})$ for every $q \geq 0$ because $\mathcal{L}^{\bullet}$ is quasi-isomorphic to $\mathcal{L}$.

We note that a stratum of $X$ means an irreducible component of $X_{i_{0}} \cap \cdots \cap X_{i_{k}}$ for some $\left\{i_{0}, \cdots, i_{k}\right\} \subset I$. If $X$ is a simple normal crossing divisor on a smooth variety $M$, then a stratum of $X$ is nothing but a $\log$ canonical center of $(M, X)$.

$\mathbf{2 . 1 5}$ (Flat base change theorem). In the proof of Theorem 1.9, we will use the flat base change theorem for relative dualizing sheaves (see [V2, $\S 3]$ and [Mo, Section 4]). We need the following statement.

Theorem 2.16. Let $f: V \rightarrow W$ be a flat projective surjective morphism from a Cohen-Macaulay quasi-projective variety $V$ to a smooth quasi-projective variety $W$. Let $g: W^{\prime} \rightarrow W$ be a finite flat morphism from a smooth quasi-projective variety $W^{\prime}$. We consider the following diagram:

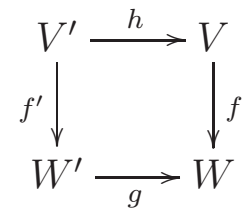

where $V^{\prime}=W^{\prime} \times_{W} V$. Then we have

$$
h^{*} \omega_{V / W}=\omega_{V^{\prime} / W^{\prime}} .
$$

Note that

$$
\omega_{V / W}=\omega_{V} \otimes f^{*} \omega_{W}^{\otimes-1} \quad \text { and } \quad \omega_{V^{\prime} / W^{\prime}}=\omega_{V^{\prime}} \otimes f^{\prime *} \omega_{W^{\prime}}^{\otimes-1} .
$$

Theorem 2.16 is a very special case of the flat base change theorem (see $[\mathrm{Vd}$, Theorem 2]). See also [H1], [Co], etc. The author does not know if the flat base change theorem ([Vd, Theorem 2]) is true or not in the analytic category (cf. $[R R]$ and $[R R V])$. Therefore, we do not 
use the flat base change theorem in the proof of Theorem 1.1 (see [V2, Lemma 3.2] and [Mo, (4.10) Base change theorem]). Note that $X$ in Theorem 1.1 is not necessarily algebraic.

2.17 (Relative vanishing theorems). The following theorem is a relative version of the Kawamata-Viehweg vanishing theorem for generically finite morphisms.

Theorem 2.18 (cf. [N1, Theorem 3.6]). Let $f: X \rightarrow Y$ be a proper generically finite morphism from a compact complex manifold $X$ onto a complex variety $Y$ and let $\Delta$ be a $\mathbb{Q}$-divisor on $X$ such that $\operatorname{Supp} \Delta$ is a simple normal crossing divisor and $\lfloor\Delta\rfloor=0$. Let $\mathcal{L}$ be a line bundle on $X$. Assume that $\mathcal{L}-\left(K_{X}+\Delta\right)$ is $f$-nef. Then $R^{i} f_{*} \mathcal{L}=0$ for every $i>0$.

Theorem 2.18 is a special case of [F7, Corollary 1.3]. For the details, see [N1], [F7], etc. Lemma 2.19, which is an easy consequence of Theorem 2.18, is very useful and indispensable.

Lemma 2.19 (Reid-Fukuda type (see [Fuk, Lemma])). Let $X$ be a compact complex manifold and let $\Delta$ be a boundary $\mathbb{Q}$-divisor on $X$ such that $\operatorname{Supp} \Delta$ is a simple normal crossing divisor on $X$. Let $f$ : $X \rightarrow Y$ be a bimeromorphic morphism onto a compact complex variety $Y$. Assume that $f$ is an isomorphism at the general points of any log canonical center of $(X, \Delta)$ and that $\mathcal{L}$ is a line bundle on $X$ such that $\mathcal{L}-\left(K_{X}+\Delta\right)$ is $f$-nef. Then $R^{i} f_{*} \mathcal{L}=0$ for every $i>0$.

Proof. By using induction on the number of irreducible components of $\lfloor\Delta\rfloor$ and on the dimension of $X$, we can quickly prove Lemma 2.19 by Theorem 2.18. For the details, see, for example, the proof of [F6, Lemma 6.2].

We close this section with a remark on the relative KawamataViehweg vanishing theorem. Anyway, the proof of Theorem 2.18 when $Y$ is not algebraic is much harder than the case when $Y$ is algebraic.

Remark 2.20 (Projective versus Kähler). We are mainly interested in projective varieties. This is because the minimal model program works well only for projective varieties. However, in this paper, we treat Kähler manifolds and complex varieties in Fujiki's class $\mathcal{C}$ in order to cover Campana's result (see [Ca, Theorem 4.13], which is essentially equivalent to Theorem 1.1). If the reader is only interested in projective varieties, then we recommend the reader to read this paper assuming that all the varieties are projective. For the minimal model program for compact Kähler manifolds and some related topics, see [CHP], [F11], [HP1], [HP2], etc. 
Let $f: X \rightarrow Y$ be a projective bimeromorphic morphism from a compact complex manifold $X$ to a compact Kähler manifold $Y$. Let $D$ be an $f$-nef Cartier divisor on $X$ such that the support of the fractional part $\{D\}$ of $D$ is a simple normal crossing divisor on $X$. Then $R^{i} f_{*} \mathcal{O}_{X}\left(K_{X}+\lceil D\rceil\right)=0$ for every $i>0$ by Theorem 2.18.

If $Y$ is projective, then the above vanishing easily follows from the usual Kawamata-Viehweg vanishing theorem for projective varieties (see [KM, Proposition 2.69] and the proof of Proposition 7.15 below). This means that the relative vanishing theorem follows from the vanishing theorem for projective varieties. On the other hand, if $Y$ is Kähler but not projective, then the above vanishing theorem is much harder to prove.

\section{Fundamental injeCtivity theOREM}

In this section, we prove Theorem 1.2. Theorem 1.2 is a direct consequence of the $E_{1}$-degeneration of Hodge to de Rham spectral sequence associated to the mixed Hodge structure for cohomology with compact support. We discuss the $E_{1}$-degeneration in Section 4 .

Proof of Theorem 1.2. Without loss of generality, we may assume that $X$ is connected. We put $S=\lfloor\Delta\rfloor$ and $B=\{\Delta\}$. By perturbing $B$, we may assume that $B$ is a $\mathbb{Q}$-divisor (see Remark 2.9). We put $\mathcal{M}=\mathcal{O}_{X}\left(\mathcal{L}-K_{X}-S\right)$. Let $N$ be the smallest positive integer such that $\mathcal{L}^{N} \sim N\left(K_{X}+S+B\right)$. In particular, $N B$ is an integral Weil divisor. We take the $N$-fold cyclic cover

$$
\pi^{\prime}: Y^{\prime}=\operatorname{Specan} \bigoplus_{i=0}^{N-1} \mathcal{M}^{-i} \rightarrow X
$$

associated to the section $N B \in\left|\mathcal{M}^{N}\right|$. More precisely, let $s \in H^{0}\left(X, \mathcal{M}^{N}\right)$ be a section whose zero divisor is $N B$. Then the dual of $s: \mathcal{O}_{X} \rightarrow \mathcal{M}^{N}$ defines an $\mathcal{O}_{X^{-}}$algebra structure on $\bigoplus_{i=0}^{N-1} \mathcal{M}^{-i}$. Let $Y \rightarrow Y^{\prime}$ be the normalization and let $\pi: Y \rightarrow X$ be the composition morphism. It is well-known that

$$
Y=\operatorname{Specan} \bigoplus_{i=0}^{N-1} \mathcal{M}^{-i}(\lfloor i B\rfloor) .
$$

For the details, see [EV, 3.5. Cyclic covers]. Note that $Y$ has only quotient singularities. We put $T=\pi^{*} S$. We note that $T$ is Cartier. Hence the locally free sheaf $\mathcal{O}_{Y}(-T)$ is the defining ideal sheaf of $T$ on $Y$. The $E_{1}$-degeneration of

$$
\text { (a) } \quad E_{1}^{p, q}=H^{q}\left(Y, \widetilde{\Omega}_{Y}^{p}(\log T)(-T)\right) \Rightarrow H^{p+q}\left(Y, j ! \mathbb{C}_{Y-T}\right) \text {, }
$$


where $j: Y-T \rightarrow Y$ is the natural open immersion, implies that the homomorphism

$$
H^{q}\left(Y, j_{!} \mathbb{C}_{Y-T}\right) \rightarrow H^{q}\left(Y, \mathcal{O}_{Y}(-T)\right)
$$

induced by the natural inclusion

$$
j_{!} \mathbb{C}_{Y-T} \subset \mathcal{O}_{Y}(-T)
$$

is surjective for every $q$. For the definition of $\widetilde{\Omega}_{Y}^{p}(\log T)(-T)$, see Definition 4.5. We will discuss the $E_{1}$-degeneration of (3) in Section 4 below. By taking a suitable direct summand

$$
\mathcal{C} \subset \mathcal{M}^{-1}(-S)
$$

of

$$
\pi_{*}\left(j_{!} \mathbb{C}_{Y-T}\right) \subset \pi_{*} \mathcal{O}_{Y}(-T),
$$

we obtain a surjection

$$
H^{q}(X, \mathcal{C}) \rightarrow H^{q}\left(X, \mathcal{M}^{-1}(-S)\right)
$$

induced by the natural inclusion $\mathcal{C} \subset \mathcal{M}^{-1}(-S)$ for every $q$. We can check the following simple property by examining the monodromy action of the Galois group $\mathbb{Z} / N \mathbb{Z}$ of $\pi: Y \rightarrow X$ on $\mathcal{C}$ around $\operatorname{Supp} B$ (see also the proof of [Ko1, 2.12.1 Proposition]).

Lemma 3.1 (cf. [KM, Corollary 2.54]). Let $U \subset X$ be a connected open subset such that $U \cap \operatorname{Supp} \Delta \neq \emptyset$. Then $H^{0}\left(U,\left.\mathcal{C}\right|_{U}\right)=0$.

Proof. If $U \cap \operatorname{Supp} B \neq \emptyset$, then $H^{0}\left(U,\left.\mathcal{C}\right|_{U}\right)=0$ since the monodromy action on $\left.\mathcal{C}\right|_{U \backslash \operatorname{Supp} B}$ around $\operatorname{Supp} B$ is nontrivial. If $U \cap \operatorname{Supp} S \neq \emptyset$, then $H^{0}\left(U,\left.\mathcal{C}\right|_{U}\right)=0$ since $\mathcal{C}$ is a direct summand of $\pi_{*}\left(j_{!} \mathbb{C}_{Y-T}\right)$ and $T=\pi^{*} S$.

Lemma 3.1 is useful by the following fact. The proof of Lemma 3.2 is obvious.

Lemma 3.2 (cf. [KM, Lemma 2.55]). Let $F$ be a sheaf of Abelian groups on a topological space $X$ and let $F_{1}$ and $F_{2}$ be subsheaves of $F$. Let $Z \subset X$ be a closed subset. Assume that

(1) $\left.F_{2}\right|_{X-Z}=\left.F\right|_{X-Z}$, and

(2) if $U \subset X$ is a connected open subset with $U \cap Z \neq \emptyset$, then $H^{0}\left(U, F_{1} \mid U\right)=0$.

Then $F_{1}$ is a subsheaf of $F_{2}$.

Therefore, we obtain: 
Corollary 3.3 (cf. [KM, Corollary 2.56]). Let $M \subset \mathcal{M}^{-1}(-S)$ be a subsheaf such that $\left.M\right|_{X-\operatorname{Supp} \Delta}=\left.\mathcal{M}^{-1}(-S)\right|_{X-\operatorname{Supp} \Delta}$. Then the injection

$$
\mathcal{C} \rightarrow \mathcal{M}^{-1}(-S)
$$

factors as

$$
\mathcal{C} \rightarrow M \rightarrow \mathcal{M}^{-1}(-S)
$$

Therefore,

$$
H^{q}(X, M) \rightarrow H^{q}\left(X, \mathcal{M}^{-1}(-S)\right)
$$

is surjective for every $q$.

Proof. The first part is clear from Lemma 3.1 and Lemma 3.2. This implies that we have maps

$$
H^{q}(X, \mathcal{C}) \rightarrow H^{q}(X, M) \rightarrow H^{q}\left(X, \mathcal{M}^{-1}(-S)\right) .
$$

As we saw above, the composition is surjective. Hence so is the map on the right.

Therefore, $H^{q}\left(X, \mathcal{M}^{-1}(-S-D)\right) \rightarrow H^{q}\left(X, \mathcal{M}^{-1}(-S)\right)$ is surjective for every $q$. By Serre duality, we obtain that

$$
H^{q}\left(X, \mathcal{O}_{X}\left(K_{X}\right) \otimes \mathcal{M}(S)\right) \rightarrow H^{q}\left(X, \mathcal{O}_{X}\left(K_{X}\right) \otimes \mathcal{M}(S+D)\right)
$$

is injective for every $q$. This means that

$$
H^{q}(X, \mathcal{L}) \rightarrow H^{q}\left(X, \mathcal{L} \otimes \mathcal{O}_{X}(D)\right)
$$

is injective for every $q$.

\section{MHS FOR COHOMOLOGY WITH COMPACT SUPPORT}

In this section, we prove the $E_{1}$-degeneration of ( $)$ in the proof of Theorem 1.2 for the reader's convenience. It is more or less well-known to the experts.

From 4.1 to 4.3, we recall some well-known results on mixed Hodge structures. We use the notations in [D] freely. The basic references on this topic are [D, Section 8], [E1, Part II], [E2, Chapitres 2 and 3], and the book [PS].

First, we start with the pure Hodge structures on complex manifolds in Fujiki's class $\mathcal{C}$.

4.1. Let $X$ be a complex manifold in Fujiki's class $\mathcal{C}$. Then the triple $\left(\mathbb{Z}_{X},\left(\Omega_{X}^{\bullet}, F\right), \alpha\right)$, where $\Omega_{X}^{\bullet}$ is the holomorphic de Rham complex with the filtration bête $F$ (see [D, (1.4.7)]) and $\alpha: \mathbb{C}_{X} \rightarrow \Omega_{X}^{\bullet}$ is the inclusion, is a cohomological Hodge complex (CHC, for short) of weight zero. 
If we define weight filtrations as follows:

$$
W_{m} \mathbb{Q}_{X}=\left\{\begin{array}{lll}
0 & \text { if } & m<0 \\
\mathbb{Q}_{X} & \text { if } & m \geq 0
\end{array}\right.
$$

and

$$
W_{m} \Omega_{X}^{\bullet}=\left\{\begin{array}{lll}
0 & \text { if } & m<0 \\
\Omega_{X}^{\bullet} & \text { if } & m \geq 0
\end{array}\right.
$$

then we can see that

$$
\left(\mathbb{Z}_{X},\left(\mathbb{Q}_{X}, W\right),\left(\Omega_{X}^{\bullet}, F, W\right)\right)
$$

is a cohomological mixed Hodge complex (CMHC, for short). We need these weight filtrations in the following arguments.

The next one is also a fundamental example. For the details, see [E1, I.1] or [E2, 3.5].

4.2. Let $D$ be a simple normal crossing variety in Fujiki's class $\mathcal{C}$. Let $\varepsilon: D^{\bullet} \rightarrow D$ be the Mayer-Vietoris simplicial resolution (see Definition 2.14). We use similar notations to those in Definition 2.14. The following complex of sheaves, denoted by $\mathbb{Q}_{D} \bullet$,

$$
\varepsilon_{0 *} \mathbb{Q}_{D^{0}} \rightarrow \varepsilon_{1 *} \mathbb{Q}_{D^{1}} \rightarrow \cdots \rightarrow \varepsilon_{k *} \mathbb{Q}_{D^{k}} \rightarrow \cdots
$$

is a resolution of $\mathbb{Q}_{D}$. More explicitly, the differential $d_{k}: \varepsilon_{k *} \mathbb{Q}_{D^{k}} \rightarrow$ $\varepsilon_{k+1 *} \mathbb{Q}_{D^{k+1}}$ is $\sum_{j=0}^{k+1}(-1)^{j} \lambda_{j, k+1}^{*}$ for every $k \geq 0$. The weight filtration $W$ on $\mathbb{Q}_{D} \cdot$ is defined by

$$
W_{-q}\left(\mathbb{Q}_{D^{\bullet}}\right)=\left(0 \rightarrow \cdots \rightarrow 0 \rightarrow \varepsilon_{q *} \mathbb{Q}_{D^{q}} \rightarrow \varepsilon_{q+1 *} \mathbb{Q}_{D^{q+1}} \rightarrow \cdots\right) .
$$

We obtain the resolution $\Omega_{D}^{\bullet}$ of $\mathbb{C}_{D}$ as follows:

$$
\varepsilon_{0 *} \Omega_{D^{0}}^{\bullet} \rightarrow \varepsilon_{1 *} \Omega_{D^{1}}^{\bullet} \rightarrow \cdots \rightarrow \varepsilon_{k *} \Omega_{D^{k}}^{\bullet} \rightarrow \cdots .
$$

Of course, $d_{k}: \varepsilon_{k *} \Omega_{D^{k}}^{\bullet} \rightarrow \varepsilon_{k+1 *} \Omega_{D^{k+1}}^{\bullet}$ is $\sum_{j=0}^{k+1}(-1)^{j} \lambda_{j, k+1}^{*}$. Let $s\left(\Omega_{D}^{\bullet}\right)$ be the single complex associated to the double complex $\Omega_{D^{\bullet}}^{\bullet}$. The Hodge filtration $F$ on $s\left(\Omega_{D^{\bullet}}^{\bullet}\right)$ is defined by

$$
F^{p}=s\left(0 \rightarrow \cdots \rightarrow 0 \rightarrow \varepsilon_{*} \Omega_{D^{\bullet}}^{p} \rightarrow \varepsilon_{*} \Omega_{D^{\bullet}}^{p+1} \rightarrow \cdots\right) .
$$

We note that

$$
\varepsilon_{*} \Omega_{D^{\bullet}}^{p}=\left(\varepsilon_{0 *} \Omega_{D^{0}}^{p} \rightarrow \varepsilon_{1 *} \Omega_{D^{1}}^{p} \rightarrow \cdots \rightarrow \varepsilon_{k *} \Omega_{D^{k}}^{p} \rightarrow \cdots\right)
$$

for every $p$. The weight filtration $W$ on $s\left(\Omega_{D^{\bullet}}^{\bullet}\right)$ is defined by

$$
W_{-q}\left(s\left(\Omega_{D^{\bullet}}^{\bullet}\right)\right)=s\left(0 \rightarrow \cdots \rightarrow 0 \rightarrow \varepsilon_{q *} \Omega_{D^{q}}^{\bullet} \rightarrow \varepsilon_{q+1 *} \Omega_{D^{q+1}}^{\bullet} \rightarrow \cdots\right) .
$$

We note that

$$
\mathrm{Gr}_{-q}^{W} \mathbb{Q}_{D^{\bullet}} \simeq \varepsilon_{q *} \mathbb{Q}_{D^{q}}[-q]
$$


and

$$
\operatorname{Gr}_{-q}^{W}\left(s\left(\Omega_{D^{\bullet}}^{\bullet}\right)\right) \simeq \varepsilon_{q^{*}} \Omega_{D^{q}}^{\bullet}[-q]
$$

Then

$$
\left(\mathbb{Z}_{D},\left(\mathbb{Q}_{D} \bullet, W\right),\left(s\left(\Omega_{D}^{\bullet}\right), W, F\right)\right)
$$

is a CMHC. Here, we omitted the quasi-isomorphisms $\alpha: \mathbb{Z}_{D} \otimes \mathbb{Q} \rightarrow$ $\mathbb{Q}_{D^{\bullet}}$ and $\beta:\left(\mathbb{Q}_{D^{\bullet}}, W\right) \otimes \mathbb{C} \rightarrow\left(s\left(\Omega_{D^{\bullet}}^{\bullet}\right), W\right)$ since there is no risk of confusion. This $\mathrm{CMHC}$ induces a natural mixed Hodge structure on $H^{\bullet}(D, \mathbb{Z})$. We note that the spectral sequence with respect to $W$ on $\mathbb{Q}_{D} \cdot$ is

$$
\begin{aligned}
{ }_{W} E_{1}^{p, q}=H^{p+q}\left(D, \mathrm{Gr}_{-p}^{W} \mathbb{Q}_{D^{\bullet}}\right) & =H^{p+q}\left(D, \varepsilon_{p *} \mathbb{Q}_{D^{p}}[-p]\right) \\
& =H^{q}\left(D^{p}, \mathbb{Q}\right) \\
& \Rightarrow H^{p+q}(D, \mathbb{Q})
\end{aligned}
$$

such that the differential $d_{1}^{p, q}:{ }_{W} E_{1}^{p, q} \rightarrow{ }_{W} E_{1}^{p+1, q}$ is given by

$$
d_{1}^{p, q}=\sum_{j=0}^{p+1}(-1)^{j} \lambda_{j, p+1}^{*}: H^{q}\left(D^{p}, \mathbb{Q}\right) \rightarrow H^{q}\left(D^{p+1}, \mathbb{Q}\right)
$$

and it degenerates at $E_{2}$. The spectral sequence with respect to $F$ is

$$
{ }_{F} E_{1}^{p, q}=\mathbb{H}^{p+q}\left(D, \operatorname{Gr}_{F}^{p}\left(s\left(\Omega_{D^{\bullet}}^{\bullet}\right)\right) \Rightarrow H^{p+q}(D, \mathbb{C})\right.
$$

and it degenerates at $E_{1}$.

For the precise definitions of CHC and CMHC (CHMC, in French), see [D, Section 8] or [E2, Chapitre 3]. See also [PS, 2.3.3 and 3.3].

The third example is not so standard but is indispensable for our injectivity theorems.

4.3. Let $X$ be a complex manifold in Fujiki's class $\mathcal{C}$ and let $D$ be a simple normal crossing divisor on $X$. We consider the mixed cones of $\phi: \mathbb{Q}_{X} \rightarrow \mathbb{Q}_{D}$ and $\psi: \Omega_{X}^{\bullet} \rightarrow \Omega_{D^{\bullet}}^{\bullet}$ with suitable shifts of complexes and weight filtrations (for the details, see, for example, [E1, I.3], [E2, 3.7.14], [EL, Section 3.3.4] or [PS, Theorem 3.22]), where $\phi$ and $\psi$ are induced by the natural restriction map. More precisely, we define a complex

$$
\mathbb{Q}_{X-D^{\bullet}}=\operatorname{Cone}^{\bullet}(\phi)[-1] .
$$

Then we have

$$
\left(\mathbb{Q}_{X-D^{\bullet}}\right)^{p}=\left(\mathbb{Q}_{X}\right)^{p} \oplus\left(\mathbb{Q}_{D} \bullet\right)^{p-1} .
$$

The weight filtration on $\mathbb{Q}_{X-D^{\bullet}}$ is defined as follows:

$$
\left(W_{m} \mathbb{Q}_{X-D^{\bullet}}\right)^{p}=\left(W_{m} \mathbb{Q}_{X}\right)^{p} \oplus\left(W_{m+1}\left(\mathbb{Q}_{D} \bullet\right)\right)^{p-1} .
$$


We note that $\mathbb{Q}_{X-D} \bullet$ is quasi-isomorphic to $j ! \mathbb{Q}_{X-D}$, where $j: X-D \rightarrow$ $X$ is the natural open immersion. We put

$$
\Omega_{X-D}^{\bullet}=\operatorname{Cone}^{\bullet}(\psi)[-1] .
$$

We note that

$$
\Omega_{X-D^{\bullet}}^{p}=\Omega_{X}^{p} \oplus\left(s \Omega_{D^{\bullet}}^{\bullet}\right)^{p-1} .
$$

We define filtrations on $\Omega_{X-D^{\bullet}}^{\bullet}$ as follows:

$$
\left(W_{m} \Omega_{X-D^{\bullet}}^{\bullet}\right)^{p}=\left(W_{m} \Omega_{X}^{\bullet}\right)^{p} \oplus\left(W_{m+1}\left(s \Omega_{D^{\bullet}}^{\bullet}\right)\right)^{p-1}
$$

and

$$
\left(F^{r} \Omega_{X-D}^{\bullet}\right)^{p}=\left(F^{r} \Omega_{X}^{\bullet}\right)^{p} \oplus\left(F^{r}\left(s \Omega_{D^{\bullet}}^{\bullet}\right)\right)^{p-1} .
$$

Then we obtain that the triple

$$
\left(j ! \mathbb{Z}_{X-D},\left(\mathbb{Q}_{X-D^{\bullet}, W},\left(\Omega_{X-D^{\bullet}}^{\bullet}, W, F\right)\right)\right.
$$

is a CMHC. It defines a natural mixed Hodge structure on $H_{c}^{\bullet}(X-$ $D, \mathbb{Z})$. We note that

$$
\operatorname{Gr}_{0}^{W} \mathbb{Q}_{X-D^{\bullet}}=\mathbb{Q}_{X}
$$

and

$$
\operatorname{Gr}_{-p}^{W} \mathbb{Q}_{X-D} \cdot=\operatorname{Gr}_{1-p}^{W} \mathbb{Q}_{D} \cdot[-1]=\varepsilon_{p-1 *} \mathbb{Q}_{D^{p-1}}[-p]
$$

for $p \geq 1$. The spectral sequence with respect to $W$

$$
{ }_{W} E_{1}^{p, q}=H^{p+q}\left(X, \operatorname{Gr}_{-p}^{W} \mathbb{Q}_{X-D} \bullet\right) \Rightarrow H_{c}^{p+q}(X-D, \mathbb{Q})
$$

degenerates at $E_{2}$, where

$$
{ }_{W} E_{1}^{0, q}=H^{q}(X, \mathbb{Q})
$$

and

$$
{ }_{W} E_{1}^{p, q}=H^{q}\left(D^{p-1}, \mathbb{Q}\right)
$$

for every $p \geq 1$. We put

$$
\Omega_{X}^{\bullet}(\log D)(-D)=\Omega_{X}^{\bullet}(\log D) \otimes \mathcal{O}_{X}(-D) .
$$

Since we can check that the complex

$$
\begin{aligned}
0 \rightarrow & \Omega_{X}^{\bullet}(\log D)(-D) \rightarrow \Omega_{X}^{\bullet} \rightarrow \varepsilon_{0 *} \Omega_{D^{0}}^{\bullet} \\
& \rightarrow \varepsilon_{1 *} \Omega_{D^{1}}^{\bullet} \rightarrow \cdots \rightarrow \varepsilon_{k *} \Omega_{D^{k}}^{\bullet} \rightarrow \cdots
\end{aligned}
$$

is exact by direct local calculations, we see that $\left(\Omega_{X-D^{\bullet}}^{\bullet}, F\right)$ is quasiisomorphic to $\left(\Omega_{X}^{\bullet}(\log D)(-D), F\right)$ in $D^{+} F(X, \mathbb{C})$, where

$$
\begin{aligned}
& F^{p} \Omega_{X}^{\bullet}(\log D)(-D) \\
& \quad=\left(0 \rightarrow \cdots \rightarrow 0 \rightarrow \Omega_{X}^{p}(\log D)(-D) \rightarrow \Omega_{X}^{p+1}(\log D)(-D) \rightarrow \cdots\right) .
\end{aligned}
$$

Therefore, the spectral sequence with respect to $F$

$$
E_{1}^{p, q}=H^{q}\left(X, \Omega_{X}^{p}(\log D)(-D)\right) \Rightarrow \mathbb{H}^{p+q}\left(X, \Omega_{X}^{\bullet}(\log D)(-D)\right)
$$


degenerates at $E_{1}$. Note that the right hand side is isomorphic to $H_{c}^{p+q}(X-D, \mathbb{C})$. We also note that

$$
\operatorname{Gr}_{F}^{0} \Omega_{X}^{\bullet}(\log D)(-D) \simeq \mathcal{O}_{X}(-D)
$$

Remark 4.4. When we take mixed cones in 4.3 , we have to be careful about the commutativity of various comparison morphisms in the derived category (see [EL, Section 3.3.4] and [PS, Remark 3.23]).

Let us recall the notion of $V$-manifolds. We need it for the proof of Theorem 1.2.

Definition 4.5 ( $V$-manifold). A $V$-manifold of dimension $d$ is a complex analytic space that admits an open covering $\left\{U_{i}\right\}$ such that each $U_{i}$ is analytically isomorphic to $V_{i} / G_{i}$, where $V_{i} \subset \mathbb{C}^{d}$ is an open ball and $G_{i}$ is a finite subgroup of $\operatorname{GL}(d, \mathbb{C})$. In this paper, $G_{i}$ is always an abelian group for every $i$.

Let $X$ be a $V$-manifold and let $\Sigma$ be its singular locus. Then we define

$$
\widetilde{\Omega}_{X}^{\bullet}=j_{*} \Omega_{X-\Sigma}^{\bullet},
$$

where $j: X-\Sigma \rightarrow X$ is the natural open immersion. A divisor $D$ on $X$ is called a divisor with $V$-normal crossings if locally on $X$ we have $(X, D) \simeq(V, E) / G$ with $V \subset \mathbb{C}^{d}$ an open domain, $G \subset \operatorname{GL}(d, \mathbb{C})$ a small subgroup acting on $V$, and $E \subset V$ a $G$-invariant normal crossing divisor. We define

$$
\widetilde{\Omega}_{X}^{\bullet}(\log D)=j_{*} \Omega_{X-\Sigma}^{\bullet}(\log D) .
$$

Furthermore, if $D$ is Cartier, then we put

$$
\widetilde{\Omega}_{X}^{\bullet}(\log D)(-D)=\widetilde{\Omega}_{X}^{\bullet}(\log D) \otimes \mathcal{O}_{X}(-D) .
$$

Let us go back to the proof of the $E_{1}$-degeneration of (3) in the proof of Theorem 1.2.

Proof of the $E_{1}$-degeneration of ( $\left.\mathbf{3}\right)$ in the proof of Theorem 1.2. Here, we use the notation in the proof of Theorem 1.2. In this case, we know that $Y$ has only quotient singularities, that is, $Y$ is a $V$-manifold. We see that $Y$ is in Fujiki's class $\mathcal{C}$ (see Remark 2.12). Then we obtain that

$$
\left(\mathbb{Z}_{Y},\left(\widetilde{\Omega}_{Y}^{\bullet}, F\right), \alpha\right)
$$

is a CHC, where $F$ is the filtration bête and $\alpha: \mathbb{C}_{Y} \rightarrow \widetilde{\Omega}_{Y}^{\bullet}$ is the inclusion. For the details, see [St, (1.6)]. By construction, $T$ is a divisor with $V$-normal crossings on $Y$ (see Definition 4.5 and [St, (1.16) Definition]). We can check that $Y$ is singular only over the singular locus of $\operatorname{Supp} B$. Let $\varepsilon: T^{\bullet} \rightarrow T$ be the Mayer-Vietoris simplicial resolution. 
Although each irreducible component of $T$ may have singularities, Definition 2.14 makes sense without any modifications. We note that $T^{n}$ has only quotient singularities for every $n \geq 0$ by the construction of $\pi: Y \rightarrow X$. We can also check that the same construction as in 4.2 works with only minor modifications. Hence we have a $\mathrm{CMHC}$

$$
\left(\mathbb{Z}_{T},\left(\mathbb{Q}_{T}^{\bullet}, W\right),\left(s\left(\widetilde{\Omega}_{T^{\bullet}}^{\bullet}\right), W, F\right)\right)
$$

that induces a natural mixed Hodge structure on $H^{\bullet}(T, \mathbb{Z})$. By the same arguments as in 4.3 , we can construct a triple

$$
\left(j ! \mathbb{Z}_{Y-T},\left(\mathbb{Q}_{Y-T} \bullet, W\right),\left(K_{\mathbb{C}}, W, F\right)\right),
$$

where $j: Y-T \rightarrow Y$ is the natural open immersion. It is a $\mathrm{CMHC}$ that induces a natural mixed Hodge structure on $H_{c}^{\bullet}(Y-T, \mathbb{Z})$ and $\left(K_{\mathbb{C}}, F\right)$ is quasi-isomorphic to $\left(\widetilde{\Omega}_{Y}^{\bullet}(\log T)(-T), F\right)$ in $D^{+} F(Y, \mathbb{C})$, where

$$
\begin{aligned}
& F^{p} \widetilde{\Omega}_{Y}^{\bullet}(\log T)(-T) \\
& \quad=\left(0 \rightarrow \cdots \rightarrow 0 \rightarrow \widetilde{\Omega}_{Y}^{p}(\log T)(-T) \rightarrow \widetilde{\Omega}_{Y}^{p+1}(\log T)(-T) \rightarrow \cdots\right) .
\end{aligned}
$$

Therefore, the spectral sequence with respect to $F$

$$
E_{1}^{p, q}=H^{q}\left(Y, \widetilde{\Omega}_{Y}^{p}(\log T)(-T)\right) \Rightarrow \mathbb{H}^{p+q}\left(Y, \widetilde{\Omega}_{Y}^{\bullet}(\log T)(-T)\right)
$$

degenerates at $E_{1}$. Note that the right hand side is isomorphic to $H_{c}^{p+q}(Y-T, \mathbb{C})=H^{p+q}\left(Y, j_{!} \mathbb{C}_{Y-T}\right)$. We also note that

$$
\operatorname{Gr}_{F}^{0} \widetilde{\Omega}_{Y}^{\bullet}(\log T)(-T) \simeq \mathcal{O}_{Y}(-T) \text {. }
$$

\section{InJECTIVITY, TORSION-FREE, AND VANISHING THEOREMS}

Once we establish Theorem 1.2, we can easily prove Theorem 1.3. Moreover, Theorem 1.4 is an easy consequence of Theorem 1.3.

Proof of Theorem 1.3. We can obtain Theorem 1.3 as an application of Theorem 1.2. More precisely, by Theorem 1.2, the proof of [F6, Theorem 6.1] works with some suitable modifications. Note that the vanishing theorem of Reid-Fukuda type for birational morphisms (see [F6, Lemma 6.2]) holds for bimeromorphic morphisms between complex varieties by Lemma 2.19. The desingularization used in the proof of [F6, Theorem 6.1] holds also for compact complex varieties (see Remark 2.5). We leave the details as exercises for the reader.

Proof of Theorem 1.4. Theorem 1.4 follows from Theorem 1.3 by some standard arguments. For (i), Step 1 in the proof of [F6, Theorem 6.3 (i)] is sufficient by Theorem 1.3 since $X$ is projective. Step 1 in the proof of [F6, Theorem 6.3 (ii)] works without any modifications by 
Theorem 1.3. Therefore, we obtain the statement (ii). For the details, see $[\mathrm{F} 6]$.

\section{Semipositivity theorem}

The following result is a special case of Theorem 1.4 (i).

Corollary 6.1. Let $X$ be a compact Kähler manifold and let $f: X \rightarrow$ $Y$ be a surjective morphism onto a projective variety $Y$. Let $D$ be a simple normal crossing divisor on $X$ such that every stratum of $D$ is dominant onto $Y$. Then $R^{i} f_{*} \mathcal{O}_{X}\left(K_{X}+D\right)$ is torsion-free for every $i$.

Once we establish Corollary 6.1, we can prove Theorem 1.5.

Proof of Theorem 1.5. By Corollary 6.1, the arguments in [F1, Section 3] work without any modifications. Therefore, we obtain Theorem 1.5 .

Remark 6.2. Theorem 1.5 is contained in [FFS]. See [FFS, 4.7. Remark]. Note that the argument in [FFS] heavily depends on Saito's theory of mixed Hodge modules.

Remark 6.3. The semipositivity of $R^{i} f_{*} \mathcal{O}_{X}\left(K_{X / Y}+D\right)$ in Theorem 1.5 follows from [FF, Theorem 1.3] or [FFS, Theorem 3]. We do not use [Kw2, Theorem 2], which depends on [Kw1]. For some comments on the semipositivity theorem in [Kw1], see [FFS, 4.6. Remark].

Remark 6.4. In Theorem 1.5, the case when $i=0$ is similar to [Kw1, Theorem 32]. Unfortunately, our results and arguments do not recover Kawamata's original statement (see [Kw1, Theorem 32]). The author has been unable to follow [Kw1, Theorem 32]. Anyway, our formulation of Theorem 1.5 is natural and the statement of Theorem 1.5 is sufficient for most applications. We do not use [Kw1, Theorem 32] in this paper. Therefore, we do not touch [Kw1, Theorem 32] here anymore. We note that $\left[\mathrm{Kw}_{\mathrm{w}}\right]$ was written before [SZ] and [Ks], where the theory of (admissible) variations of mixed Hodge structure was investigated.

Remark 6.5 (On canonical extensions). In the last paragraph in [Kw3, Section 2], Yujiro Kawamata wrote:

The Hodge filtration $F$ of $\mathcal{H}$ extends to $\tilde{\mathcal{H}}$ such that $\operatorname{Gr}_{F}(\tilde{\mathcal{H}})$ is still a locally free sheaf on $\tilde{Y}$. Indeed this is a consequence of the nilpotent orbit theorem [14] when $H$ is a variation of pure Hodge structures, and the general case follows immediately from this. 
Note that [14] is Schmid's famous paper: [Schm]. Kawamata's statement is obviously wrong when $H$ is a variation of mixed Hodge structure. In [Kw4, 5. Canonical extension], he also wrote:

By [17], the Hodge filtration of $\mathcal{H}$ extends to a filtration by locally free subsheaves, which we denote again by $F$.

We note that [17] is Schmid's paper: [Schm].

As mentioned above, in [Kw3] and [Kw4], Yujiro Kawamata seems to misuse Schmid's nilpotent orbit theorem. It is a result for polarizable variations of pure Hodge structure. We can not directly apply it to graded polarizable variations of mixed Hodge structure. For some explicit examples on this topic, see [FF, Example 1.5], [SZ, (3.16) Example], etc. Therefore, we do not use the results in [Kw3] and [Kw4] in this paper.

Note that the main theorem of [FF] (see [FF, Theorem 1.1]), whose proof is completely different from Kawamata's argument in [Kw3], is sharper than Kawamata's main statement in [Kw3] (see [Kw3, Theorem 1.1]). We also note that [Kw3, Theorem 1.1] does not seem to cover the semipositivity theorem in [F1] directly (see [F1, Theorem 3.9]). This is because [Kw3, Theorem 1.1] requires some extra assumptions on every stratum. Roughly speaking, [F1, Theorem 3.9] is Theorem 1.5 under the assumption that $X$ is a smooth projective variety.

Anyway, we have established Theorem 1.5, which is the main ingredient of the twisted weak positivity: Theorem 1.1.

\section{WEAKLY POSITIVE SHEAVES}

In this section, we discuss weakly positive sheaves introduced by Viehweg (see [V1] and [V2]). For the basic properties of weakly positive sheaves and related results, see, for example, [V4, Section 2] and [F15]. In this paper, we closely follow [V2], [V4, Section 2], [Ca, Section 4.4], and [Mo]. Here, we adopt [V3, Definition 3.1] for the definition of weakly positive sheaves, which is slightly different from Viehweg's original definition (see [V1] and [V2, Definition 1.2]).

Definition 7.1. Let $W$ be a smooth projective variety and let $\mathcal{F}$ be a torsion-free coherent sheaf on $W$. Let $\widehat{W}$ be the largest Zariski open subset of $W$ such that $\left.\mathcal{F}\right|_{\widehat{W}}$ is locally free. Then we put

$$
\widehat{S}^{k}(\mathcal{F})=i_{*} S^{k}\left(i^{*} \mathcal{F}\right)
$$

where $i: \widehat{W} \rightarrow W$ is the natural open immersion and $S^{k}$ denotes the $k$-th symmetric product. Note that $\operatorname{codim}_{W}(W \backslash \widehat{W}) \geq 2$ since $\mathcal{F}$ is torsion-free. 
Definition 7.2 (Weak positivity). Let $W$ be a smooth projective variety and let $\mathcal{F}$ be a torsion-free coherent sheaf on $W$. We call $\mathcal{F}$ weakly positive, if for every ample line bundle $\mathcal{H}$ on $W$ and every positive integer $\alpha$ there exists some positive integer $\beta$ such that $\widehat{S}^{\alpha \beta}(\mathcal{F}) \otimes \mathcal{H}^{\otimes \beta}$ is generically generated by global sections. This means that the natural map

$$
H^{0}\left(W, \widehat{S}^{\alpha \beta}(\mathcal{F}) \otimes \mathcal{H}^{\otimes \beta}\right) \otimes \mathcal{O}_{W} \rightarrow \widehat{S}^{\alpha \beta}(\mathcal{F}) \otimes \mathcal{H}^{\otimes \beta}
$$

is generically surjective. By definition, the trivial sheaf $\mathcal{F}=0$ is weakly positive.

In some literature, the following definition is used for weakly positive sheaves on smooth projective varieties (see, for example, [V1], and [V2, Definition 1.2]).

Definition 7.3 (Original weak positivity). Let $W$ be a smooth projective variety and let $\mathcal{F}$ be a torsion-free coherent sheaf on $W$. Let $U$ be a Zariski open set of $W$. We call $\mathcal{F}$ weakly positive over $U$, if for every ample line bundle $\mathcal{H}$ on $W$ and every positive integer $\alpha$ there exists some positive integer $\beta$ such that $\widehat{S}^{\alpha \beta}(\mathcal{F}) \otimes \mathcal{H}^{\otimes \beta}$ is generated by global sections over $U$. This means that the natural map

$$
H^{0}\left(W, \widehat{S}^{\alpha \beta}(\mathcal{F}) \otimes \mathcal{H}^{\otimes \beta}\right) \otimes \mathcal{O}_{W} \rightarrow \widehat{S}^{\alpha \beta}(\mathcal{F}) \otimes \mathcal{H}^{\otimes \beta}
$$

is surjective over $U$. We call $\mathcal{F}$ weakly positive, if there exists some nonempty Zariski open set $U$ such that $\mathcal{F}$ is weakly positive over $U$.

Note that $U$ is independent of $\alpha$ and $\beta$ in Definition 7.3. It is obvious that if $\mathcal{F}$ is weakly positive in the sense of Definition 7.3 then $\mathcal{F}$ is weakly positive in the sense of Definition 7.2.

In this paper, we do not use the weak positivity in the sense of Definition 7.3, which is slightly stronger but is harder to prove than the weak positivity in the sense of Definition 7.2.

Remark 7.4. Let $W$ and $\mathcal{F}$ be as in Definition 7.2. By Definition 7.2, $\mathcal{F}$ is weakly positive if and only if so is $\widehat{S}^{1}(\mathcal{F})=\mathcal{F}^{* *}$, where $\mathcal{F}^{* *}$ is the double-dual of $\mathcal{F}$.

Remark 7.5 (Nef locally free sheaf). Let $W$ be a smooth projective variety and let $\mathcal{F}$ be a locally free sheaf of finite rank on $W$. If $\mathcal{F}$ is nef, that is, $\mathcal{F}=0$ or $\mathcal{O}_{\mathbb{P}_{W}(\mathcal{F})}(1)$ is a nef line bundle on $\mathbb{P}_{W}(\mathcal{F})$, then $\mathcal{F}$ is weakly positive.

More precisely, let $\mathcal{F} \neq 0$ be a nef locally free sheaf of finite rank on a smooth projective variety $W$ and let $\mathcal{H}$ be an ample line bundle on $W$. We put $\pi: \mathbb{P}_{W}(\mathcal{F}) \rightarrow W$. Then we can easily check that $\mathcal{O}_{\mathbb{P}_{W}(\mathcal{F})}(\alpha) \otimes \pi^{*} \mathcal{H}$ is an ample line bundle for every positive integer $\alpha$ by 
Nakai's criterion. In particular, $\mathcal{O}_{\mathbb{P}_{W}(\mathcal{F})}(1) \otimes \pi^{*} \mathcal{H}$ is ample, equivalently, $\mathcal{F} \otimes \mathcal{H}$ is ample. By the argument b) in the proof of [H2, Proposition (3.2)], there is a positive integer $\beta_{0}$ such that $S^{\alpha \beta}(\mathcal{F}) \otimes \mathcal{H}^{\otimes \beta}$ is generated by global sections for every integer $\beta \geq \beta_{0}$. Therefore, $\mathcal{F}$ is weakly positive.

Remark 7.6. Let $\mathcal{F}$ be a line bundle on a smooth projective variety $W$. Then $\mathcal{F}$ is weakly positive if and only if $\mathcal{F}$ is pseudo-effective.

Although Lemma 7.7 is obvious by the definition of weakly positive sheaves, it is very useful. We will repeatedly use Lemma 7.7 in this section.

Lemma 7.7 (cf. [V2, Lemma 1.4. 1)]). Let $W$ be a smooth projective variety and let $\mathcal{F}$ and $\mathcal{G}$ be torsion-free coherent sheaves on $W$. If $\mathcal{F} \rightarrow \mathcal{G}$ is a morphism which is generically surjective and if $\mathcal{F}$ is weakly positive, then $\mathcal{G}$ is also weakly positive.

Let us prove the following generalization of Viehweg's theorem (cf. [V2, Theorem 4.1]), which follows from Theorem 1.5. Viehweg only considered the case when $i=0$ with $D=0$.

Theorem 7.8 (Fundamental weak positivity theorem). Let $f: V \rightarrow$ $W$ be a surjective morphism from a compact Kähler manifold $V$ to a smooth projective variety $W$. Let $D$ be a simple normal crossing divisor on $V$ such that every irreducible component of $D$ is dominant onto $W$. Let $\Sigma$ be a simple normal crossing divisor on $W$ such that $f$ is smooth over $W_{0}=W \backslash \Sigma$ and that $D$ is relatively normal crossing over $W_{0}$, and $\operatorname{Supp}\left(D+f^{*} \Sigma\right)$ is a simple normal crossing divisor on $V$. Then the locally free sheaf $R^{i} f_{*} \mathcal{O}_{V}\left(K_{V / W}+D\right)$ is weakly positive for every $i \geq 0$.

Proof. We put $V_{0}=f^{-1}\left(W_{0}\right), f_{0}=\left.f\right|_{V_{0}}, D_{0}=\left.D\right|_{V_{0}}$, and $d=\operatorname{dim} V-$ $\operatorname{dim} W$. We take a finite morphism $g: W^{\prime} \rightarrow W$ from a smooth projective variety, which induces a unipotent reduction for the local system $R^{d+i} f_{0 *} \mathbb{C}_{V_{0}-D_{0}}$, such that $\operatorname{Supp}\left(g^{*} \Sigma\right)$ is a simple normal crossing divisor on $W^{\prime}$ (see, for example, [KM, Proposition 2.67] and [Kw1, Theorem 17]). We can construct a commutative diagram:

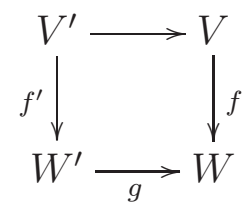

with the following properties: 
(i) $V^{\prime}$ is a compact Kähler manifold which is a resolution of $V \times_{W}$ $W^{\prime}$

(ii) $f^{\prime}$ is smooth over $W_{0}^{\prime}=W^{\prime} \backslash \Sigma^{\prime}$, where $\Sigma^{\prime}=\operatorname{Supp}\left(g^{*} \Sigma\right)$,

(iii) $D^{\prime}$ is a simple normal crossing divisor on $V^{\prime}$ such that $D^{\prime}$ and $f^{\prime *} \Sigma^{\prime}$ have no common irreducible components and that $\operatorname{Supp}\left(D^{\prime}+f^{\prime *} \Sigma^{\prime}\right)$ is a simple normal crossing divisor on $V^{\prime}$, and

(iv) $f^{\prime}:\left(V^{\prime}, D^{\prime}\right) \rightarrow W^{\prime}$ is nothing but the base change of $f$ : $(V, D) \rightarrow W$ over $W_{0}^{\prime}$.

Then we obtain a natural inclusion of locally free sheaves

$$
\varphi^{i}: R^{i} f_{*}^{\prime} \mathcal{O}_{V^{\prime}}\left(K_{V^{\prime} / W^{\prime}}+D^{\prime}\right) \subset g^{*} R^{i} f_{*} \mathcal{O}_{V}\left(K_{V / W}+D\right) .
$$

such that $\varphi^{i}$ is the identity over $W_{0}^{\prime}$. Note that $R^{i} f_{*} \mathcal{O}_{V}\left(K_{V / W}+D\right)$ is the upper canonial extension of the bottom Hodge filtration and that $R^{i} f_{*}^{\prime} \mathcal{O}_{V^{\prime}}\left(K_{V^{\prime} / W^{\prime}}+D^{\prime}\right)$ is the canonical extension of the bottom Hodge filtration (see Theorem 1.5). By Theorem 1.5, $R^{i} f_{*}^{\prime} \mathcal{O}_{V^{\prime}}\left(K_{V^{\prime} / W^{\prime}}+D^{\prime}\right)$ is nef. In particular, $R^{i} f_{*}^{\prime} \mathcal{O}_{V^{\prime}}\left(K_{V^{\prime} / W^{\prime}}+D^{\prime}\right)$ is weakly positive (see Remark 7.5). Let $H$ be an ample Cartier divisor on $W$ and let $\alpha$ be a positive integer. Then

$$
S^{2 \alpha \beta}\left(g^{*} R^{i} f_{*} \mathcal{O}_{V}\left(K_{V / W}+D\right)\right) \otimes \mathcal{O}_{W^{\prime}}\left(\beta g^{*} H\right)
$$

is generically generated by global sections for some positive integer $\beta$ since $g^{*} R^{i} f_{*} \mathcal{O}_{V}\left(K_{V / W}+D\right)$ is weakly positive by Lemma 7.7. Without loss of generality, we may assume that $\mathcal{O}_{W}(\beta H) \otimes g_{*} \mathcal{O}_{W^{\prime}}$ is generated by global sections. We have a surjection

$$
g_{*} g^{*} S^{2 \alpha \beta}\left(R^{i} f_{*} \mathcal{O}_{V}\left(K_{V / W}+D\right)\right) \rightarrow S^{2 \alpha \beta}\left(R^{i} f_{*} \mathcal{O}_{V}\left(K_{V / W}+D\right)\right) .
$$

Therefore, we have a generically surjective homomorphism

$$
\bigoplus_{\text {finite }} \mathcal{O}_{W}(\beta H) \otimes g_{*} \mathcal{O}_{W^{\prime}} \rightarrow S^{2 \alpha \beta}\left(R^{i} f_{*} \mathcal{O}_{V}\left(K_{V / W}+D\right)\right) \otimes \mathcal{O}_{W}(2 \beta H) .
$$

Thus, we obtain that

$$
S^{2 \alpha \beta}\left(R^{i} f_{*} \mathcal{O}_{V}\left(K_{V / W}+D\right)\right) \otimes \mathcal{O}_{W}(2 \beta H)
$$

is generically generated by global sections. This means that the locally free sheaf $R^{i} f_{*} \mathcal{O}_{V}\left(K_{V / W}+D\right)$ is weakly positive.

Remark 7.9. In general, the locally free sheaf $R^{i} f_{*} \mathcal{O}_{V}\left(K_{V / W}+D\right)$ is not necessarily nef. See [FF, Section 8] for some examples.

Remark 7.10. In Theorem 7.8, it is easy to see that $R^{i} f_{*} \mathcal{O}_{V}\left(K_{V / W}+\right.$ $D)$ is weakly positive over $W_{0}$ in the sense of Definition 7.3.

By using the basic properties of weakly positive sheaves, we can obtain the following corollary of Theorem 7.8, which is new when $i>0$. 
Corollary 7.11. Let $f: V \rightarrow W$ be a surjective morphism from a compact Kähler manifold $V$ to a smooth projective variety $W$. Let $D$ be a simple normal crossing divisor on $V$. Then the torsion-free part of $R^{i} f_{*} \mathcal{O}_{V}\left(K_{V / W}+D\right)$, that is,

$$
R^{i} f_{*} \mathcal{O}_{V}\left(K_{V / W}+D\right) / \text { torsion, }
$$

is weakly positive.

Proof. By replacing $D$ with its horizontal part, we may assume that every irreducible component of $D$ is dominant onto $W$ (see Lemma 7.7). If there is a log canonical center $C$ of $(V, D)$ such that $f(C) \subsetneq W$, then we take the blow-up $h: V^{\prime} \rightarrow V$ along $C$. We put

$$
K_{V^{\prime}}+D^{\prime}=h^{*}\left(K_{V}+D\right) \text {. }
$$

Then $D^{\prime}$ is a simple normal crossing divisor on $V^{\prime}$ and

$$
R^{i} f_{*} \mathcal{O}_{V}\left(K_{V / W}+D\right) \simeq R^{i}(f \circ h)_{*} \mathcal{O}_{V^{\prime}}\left(K_{V^{\prime} / W}+D^{\prime}\right)
$$

for every $i$. Therefore, we can replace $(V, D)$ with $\left(V^{\prime}, D^{\prime}\right)$. Then we replace $D$ with its horizontal part (see Lemma 7.7). By repeating this process finitely many times, we may assume that every stratum of $D$ is dominant onto $W$. In this case, $R^{i} f_{*} \mathcal{O}_{V}\left(K_{V / W}+D\right)$ is torsion-free by Theorem 1.4 (i). Now we take a closed subset $\Sigma$ of $W$ such that $f$ is smooth over $W \backslash \Sigma$ and that $D$ is relatively normal crossing over $W \backslash \Sigma$. Let $g: W^{\prime} \rightarrow W$ be a birational morphism from a smooth projective variety $W^{\prime}$ such that $\Sigma^{\prime}=g^{-1}(\Sigma)$ is a simple normal crossing divisor. By taking some suitable blow-ups of $V$ in $f^{-1}(\Sigma)$ and replacing $D$ with its strict transform, we may further assume the following conditions:

(i) $f^{\prime}=g^{-1} \circ f: V \rightarrow W^{\prime}$ is a morphism,

(ii) $f^{\prime}$ is smooth over $W^{\prime} \backslash \Sigma^{\prime}$ and $D$ is relatively normal crossing over $W^{\prime} \backslash \Sigma^{\prime}$, and

(iii) every irreducible component of $D$ is dominant onto $W$ and $\operatorname{Supp}\left(f^{\prime *} \Sigma^{\prime}+D\right)$ is a simple normal crossing divisor on $V$.

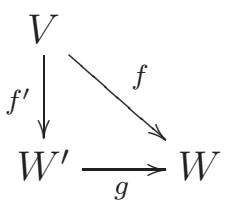

Here we used Szabó's resolution lemma (see Remark 2.5) and Lemma 2.19. Then, by Theorem $7.8, R^{i} f_{*}^{\prime} \mathcal{O}_{V}\left(K_{V / W^{\prime}}+D\right)$ is weakly positive. Note that

$$
R^{i} f_{*}^{\prime} \mathcal{O}_{V}\left(K_{V / W}+D\right) \simeq R^{i} f_{*}^{\prime} \mathcal{O}_{V}\left(K_{V / W^{\prime}}+D\right) \otimes \mathcal{O}_{W^{\prime}}(E)
$$


where $E$ is a $g$-exceptional effective divisor such that $K_{W^{\prime}}=g^{*} K_{W}+E$. Thus $R^{i} f_{*}^{\prime} \mathcal{O}_{V}\left(K_{V / W}+D\right)$ is weakly positive. We note that

$$
g_{*} R^{i} f_{*}^{\prime} \mathcal{O}_{V}\left(K_{V / W}+D\right) \simeq R^{i} f_{*} \mathcal{O}_{V}\left(K_{V / W}+D\right) .
$$

Here we used the fact that

$$
R^{p} g_{*} R^{q} f_{*}^{\prime} \mathcal{O}_{V}\left(K_{V / W}+D\right)=0
$$

for every $p>0$ and $q \geq 0$ by Proposition 7.15 below. We can take an effective $g$-exceptional divisor $F$ on $W^{\prime}$ such that $-F$ is $g$-ample. Let $H$ be an ample Cartier divisor on $W$. Then there exists a positive integer $k$ such that $k g^{*} H-F$ is ample. Since $R^{i} f_{*}^{\prime} \mathcal{O}_{V}\left(K_{V / W}+D\right)$ is weakly positive,

$$
S^{k \alpha \beta}\left(R^{i} f_{*}^{\prime} \mathcal{O}_{V}\left(K_{V / W}+D\right)\right) \otimes \mathcal{O}_{W^{\prime}}\left(\beta\left(k g^{*} H-F\right)\right)
$$

is generically generated by global sections. By taking $g_{*}$,

$$
\widehat{S}^{\alpha k \beta}\left(R^{i} f_{*} \mathcal{O}_{V}\left(K_{V / W}+D\right)\right) \otimes \mathcal{O}_{W}(k \beta H)
$$

is generically generated by global sections. This means that the torsionfree sheaf $R^{i} f_{*} \mathcal{O}_{V}\left(K_{V / W}+D\right)$ is weakly positive.

Remark 7.12. In the proof of Corollary 7.11, the following isomorphism

$$
\left(g_{*} R^{i} f_{*}^{\prime} \mathcal{O}_{V}\left(K_{V / W}+D\right)\right)^{* *} \simeq\left(R^{i} f_{*} \mathcal{O}_{V}\left(K_{V / W}+D\right)\right)^{* *}
$$

is obvious since $g$ is birational. This isomorphism is sufficient for the proof of the weak positivity of $R^{i} f_{*} \mathcal{O}_{V}\left(K_{V / W}+D\right)$ (see Remark 7.4) although we used a shaper isomorphism

$$
g_{*} R^{i} f_{*}^{\prime} \mathcal{O}_{V}\left(K_{V / W}+D\right) \simeq R^{i} f_{*} \mathcal{O}_{V}\left(K_{V / W}+D\right) .
$$

Remark 7.13. In Corollary 7.11, we take a Zariski open set $W_{0}$ of $W$ such that $f$ is smooth over $W_{0}$ and that $D$ is relatively normal crossing over $W_{0}$. Then we see that

$$
R^{i} f_{*} \mathcal{O}_{V}\left(K_{V / W}+D\right) / \text { torsion }
$$

is weakly positive over $W_{0}$ in the sense of Definition 7.3 by the proof of Corollary 7.11.

Before we give a proof of Proposition 7.15, we prepare an easy lemma.

Lemma 7.14. Theorem 1.4 (ii) holds true even when $Y$ is a complex manifold in Fujiki's class $\mathcal{C}$ and $\Delta$ is an effective $\mathbb{R}$-divisor on $Y$ such that $(Y, \Delta)$ is dlt. 
Proof. Let $h: Y^{\prime} \rightarrow Y$ be a resolution such that $h$ is an isomorphism over the general points of any log canonical center of $(Y, \Delta)$ (see Remark 2.5). We can write

$$
K_{Y^{\prime}}+\Delta_{Y^{\prime}}=h^{*}\left(K_{Y}+\Delta\right)+E
$$

where $\Delta_{Y^{\prime}}$ and $E$ are effective, $E$ is Cartier and $h$-exceptional, $\Delta_{Y^{\prime}}$ is a boundary $\mathbb{R}$-divisor, and $\operatorname{Supp}\left(\Delta_{Y^{\prime}}+E\right)$ is a simple normal crossing divisor on $Y^{\prime}$. Then

$$
\begin{aligned}
h^{*} \mathcal{L} \otimes \mathcal{O}_{Y^{\prime}}(E)-\left(K_{Y^{\prime}}+\Delta_{Y^{\prime}}\right) & =h^{*}\left(\mathcal{L}-\left(K_{Y}+\Delta\right)\right) \\
& \sim_{\mathbb{R}} h^{*} f^{*} H .
\end{aligned}
$$

By Theorem 1.4 (ii), we obtain that

$$
H^{p}\left(X, R^{q}(f \circ h)_{*}\left(h^{*} \mathcal{L} \otimes \mathcal{O}_{Y^{\prime}}(E)\right)\right)=0
$$

for every $p>0$ and $q \geq 0$. Note that $R^{i} h_{*}\left(h^{*} \mathcal{L} \otimes \mathcal{O}_{Y^{\prime}}(E)\right)=0$ for every $i>0$ by Lemma 2.19. Thus we obtain

$$
H^{p}\left(X, R^{q} f_{*} \mathcal{L}\right) \simeq H^{p}\left(X, R^{q}(f \circ h)_{*}\left(h^{*} \mathcal{L} \otimes \mathcal{O}_{Y^{\prime}}(E)\right)\right)=0
$$

for every $p>0$ and $q \geq 0$. Note that $h_{*}\left(h^{*} \mathcal{L} \otimes \mathcal{O}_{Y^{\prime}}(E)\right) \simeq \mathcal{L}$.

Proposition 7.15. Let $f: X \rightarrow Y$ be a surjective morphism such that $X$ is a complex manifold in Fujiki's class $\mathcal{C}$ and $Y$ is a projective variety. Let $D$ be a simple normal crossing divisor on $X$ such that every stratum of $D$ is dominant onto $Y$. Let $g: Y \rightarrow Z$ be a birational morphism between projective varieties. Then

$$
R^{p} g_{*} R^{q} f_{*} \mathcal{O}_{X}\left(K_{X}+D\right)=0
$$

for every $p>0$ and $q \geq 0$.

Proof. Let $\mathcal{A}$ be a sufficiently ample line bundle on $Z$ such that

$$
H^{r}\left(Z, R^{p} g_{*} R^{q} f_{*} \mathcal{O}_{X}\left(K_{X}+D\right) \otimes \mathcal{A}\right)=0
$$

for $p>0, q \geq 0$, and $r>0$ and that

$$
R^{p} g_{*} R^{q} f_{*} \mathcal{O}_{X}\left(K_{X}+D\right) \otimes \mathcal{A}
$$

is generated by global sections for $p>0$ and $q \geq 0$. By the Leray spectral sequence, we have

$$
\begin{aligned}
& H^{0}\left(Z, R^{p} g_{*} R^{q} f_{*} \mathcal{O}_{X}\left(K_{X}+D\right) \otimes \mathcal{A}\right) \\
& \simeq H^{p}\left(Y, R^{q} f_{*} \mathcal{O}_{X}\left(K_{X}+D+f^{*} g^{*} \mathcal{A}\right)\right) .
\end{aligned}
$$

Therefore, it is sufficient to prove that

$$
H^{p}\left(Y, R^{q} f_{*} \mathcal{O}_{X}\left(K_{X}+D+f^{*} g^{*} \mathcal{A}\right)\right)=0
$$


for $p>0$ and $q \geq 0$. By Kodaira's lemma, we can write

$$
g^{*} \mathcal{A} \sim_{\mathbb{Q}} H+E
$$

where $H$ is an ample $\mathbb{Q}$-divisor on $Y$ and $E$ is an effective $\mathbb{Q}$-Cartier $\mathbb{Q}$-divisor on $Y$. Let $\varepsilon$ be a sufficiently small positive number. Then $\left(X, D+\varepsilon f^{*} E\right)$ is dlt and

$$
\mathcal{O}_{X}\left(K_{X}+D+f^{*} g^{*} \mathcal{A}\right)-\left(K_{X}+D+\varepsilon f^{*} E\right) \sim_{\mathbb{Q}} f^{*}\left((1-\varepsilon) g^{*} \mathcal{A}+\varepsilon H\right) .
$$

Therefore, by Lemma 7.14, we obtain that

$$
H^{p}\left(Y, R^{q} f_{*} \mathcal{O}_{X}\left(K_{X}+D+f^{*} g^{*} \mathcal{A}\right)\right)=0
$$

for $p>0$ and $q \geq 0$.

\section{TWISTED WEAK POSITIVITY}

This section is the main part of this paper. Here, we prove the twisted weak positivity theorem: Theorem 1.1 .

Lemma 8.1 is a slight generalization of [V2, Lemma 5.1]. It follows from Corollary 7.11 by the usual covering trick.

Lemma 8.1 (cf. [V2, Lemma 5.1]). Let $f: X \rightarrow Y$ be a surjective morphism from a compact Kähler manifold $X$ to a smooth projective variety $Y$. Let $D$ be a simple normal crossing divisor on $X$. Let $\mathcal{L}$ and $\mathcal{N}$ be line bundles on $X$ and let $C$ be an effective divisor on $X$ such that $\mathcal{L}^{N}=\mathcal{N}+C$ for some positive integer $N, D$ and $C$ have no common components, and $\operatorname{Supp}(D+C)$ is a simple normal crossing divisor on $X$. Assume that there is a nonempty Zariski open set $U$ of $Y$ such that some power of $\mathcal{N}$ is generated over $f^{-1}(U)$ by global sections. Then the sheaf

$$
f_{*} \mathcal{O}_{X}\left(K_{X / Y}+D+\mathcal{L}^{(i)}\right)
$$

is weakly positive for $0 \leq i \leq N-1$, where

$$
\mathcal{L}^{(i)}=\mathcal{L}^{i} \otimes \mathcal{O}_{X}\left(-\left\lfloor\frac{i C}{N}\right\rfloor\right) .
$$

Proof. Since the statement is compatible with replacing $N$ by $N N^{\prime}, C$ by $N^{\prime} C$, and $\mathcal{N}$ by $\mathcal{N}^{N^{\prime}}$ for some positive integer $N^{\prime}$, we may assume that $\mathcal{N}$ itself is generated by global sections over $f^{-1}(U)$. This means that the base locus of $|\mathcal{N}|$ is contained in $X \backslash f^{-1}(U)$. Without loss of generality, we may shrink $U$ if necessary. Let $B+F$ be the zero set of a general section of $\mathcal{N}$ such that every irreducible component of $B$ is dominant onto $Y$ and that $\operatorname{Supp} F \subset X \backslash f^{-1}(U)$. By Bertini's theorem, $B$ is smooth and $\operatorname{Supp}(B+D+C)$ is a simple normal crossing divisor on $f^{-1}(U)$. We note that $\mathcal{N}=\mathcal{O}_{X}(B+F)$. By taking a suitable bimeromorphic modification outside $f^{-1}(U)$, we may assume that $B$ is 
smooth and that $\operatorname{Supp}(B+D+C+F)$ is a simple normal crossing divisor (see Remark 2.5). In fact, if $h: \widetilde{X} \rightarrow X$ is a bimeromorphic modification which is an isomorphism over $f^{-1}(U)$ and if $\widetilde{\mathcal{L}}=h^{*} \mathcal{L}$, $\widetilde{\mathcal{N}}=h^{*} \mathcal{N}, \widetilde{C}=h^{*} C$, and $\widetilde{D}$ is the strict transform of $D$, then we can easily check that $h_{*} \mathcal{O}_{\widetilde{X}}\left(K_{\widetilde{X} / Y}+\widetilde{D}+\widetilde{\mathcal{L}}^{(i)}\right)$ is contained in $\mathcal{O}_{X}\left(K_{X / Y}+\right.$ $\left.D+\mathcal{L}^{(i)}\right)$. By construction, $h_{*} \mathcal{O}_{\widetilde{X}}\left(K_{\widetilde{X} / Y}+\widetilde{D}+\widetilde{\mathcal{L}}^{(i)}\right)$ coincides with $\mathcal{O}_{X}\left(K_{X / Y}+D+\mathcal{L}^{(i)}\right)$ on $f^{-1}(U)$. When we prove the weak positivity of $f_{*} \mathcal{O}_{X}\left(K_{X / Y}+D+\mathcal{L}^{(i)}\right)$, by replacing $C$ with $C+F$, we may assume that $\mathcal{L}^{N}=\mathcal{O}_{X}(B+C)$, that is, $F=0$ (see Lemma 7.7). Note that every irreducible component of $F$ is vertical with respect to $f: X \rightarrow Y$. By taking a cyclic cover $p: Z^{\prime} \rightarrow X$ associated to $\mathcal{L}^{N}=\mathcal{O}_{X}(B+C)$, that is, $Z^{\prime}$ is the normalization of Specan $\bigoplus_{i=1}^{N-1} \mathcal{L}^{-i}$. Let $Z$ be a resolution of the cyclic cover $Z^{\prime}$ and let $g: Z \rightarrow Y$ be the corresponding morphism.

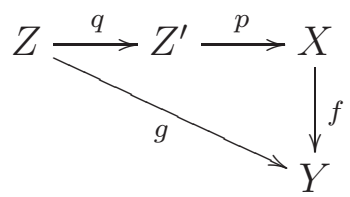

It is well-known that $Z^{\prime}$ has only quotient singularities and

$$
p_{*} \mathcal{O}_{Z^{\prime}}\left(K_{Z^{\prime}}\right) \simeq \bigoplus_{i=0}^{N-1} \mathcal{O}_{X}\left(K_{X}+\mathcal{L}^{(i)}\right) .
$$

Let $D^{\dagger}$ be the union of the strict transform of $p^{*} D$ and the exceptional divisor of $q: Z \rightarrow Z^{\prime}$. Then

$$
q_{*} \mathcal{O}_{Z}\left(K_{Z}+D^{\dagger}\right) \simeq \mathcal{O}_{Z^{\prime}}\left(K_{Z^{\prime}}+p^{*} D\right) \text {. }
$$

Note that $\left(Z^{\prime}, p^{*} D\right)$ is log canonical. Of course, we may assume that $D^{\dagger}$ is a simple normal crossing divisor. We obtain

$$
g_{*} \mathcal{O}_{Z}\left(K_{Z / Y}+D^{\dagger}\right) \simeq \bigoplus_{i=0}^{N-1} f_{*} \mathcal{O}_{X}\left(K_{X / Y}+D+\mathcal{L}^{(i)}\right)
$$

Therefore, by Corollary $7.11, f_{*} \mathcal{O}_{X}\left(K_{X / Y}+D+\mathcal{L}^{(i)}\right)$ is weakly positive for every $i$.

Before we start the proof of Theorem 1.1, we prepare a very important lemma.

Lemma 8.2 (cf. [Ca, Lemma 4.19]). Let $f: X \rightarrow Y$ be a surjective morphism from a compact Kähler manifold $X$ to a smooth projective variety $Y$. Let $\Delta$ be a boundary $\mathbb{Q}$-divisor on $X$ such that $\operatorname{Supp} \Delta$ is a simple normal crossing divisor. Let $l$ be a positive integer such that 
$l\left(K_{X / Y}+\Delta\right)$ is Cartier. Let $A^{\prime}$ be an ample Cartier divisor on $Y$. We put $A=f^{*} A^{\prime}$. Assume that

$$
\widehat{S}^{N}\left(f_{*} \mathcal{O}_{X}\left(l\left(K_{X / Y}+\Delta\right)+l A\right)\right)
$$

is generated by global sections on some nonempty Zariski open set of $Y$. Then

$$
f_{*} \mathcal{O}_{X}\left(l\left(K_{X / Y}+\Delta\right)+(l-1) A\right)
$$

is weakly positive.

Proof. We consider

$$
\mathcal{M}:=\operatorname{Im}\left(f^{*} f_{*} \mathcal{O}_{X}\left(l\left(K_{X / Y}+\Delta\right)+l A\right) \rightarrow \mathcal{O}_{X}\left(l\left(K_{X / Y}+\Delta\right)+l A\right)\right) .
$$

We may assume that the relative base locus of $l\left(K_{X / Y}+\Delta\right)+l A$ does not contain any component of $l \Delta$ by decreasing the relevant coefficients of $\Delta$. Furthermore, if necessary, by taking blow-ups of $X$ and decreasing the relevant coefficients of $\Delta$, we may assume that $\mathcal{M}$ is a line bundle, $l\left(K_{X / Y}+\Delta\right)+l A=\mathcal{M}+E$, where $E$ is an effective divisor on $X, E$ and $l \Delta$ have no common components, and $\operatorname{Supp}(E+\Delta)$ is a simple normal crossing divisor on $X$. Let

$$
f: X \stackrel{\psi}{\longrightarrow} V \longrightarrow Y
$$

be the Stein factorization. Then

$$
\mathcal{M}=\operatorname{Im}\left(\psi^{*} \psi_{*} \mathcal{O}_{X}\left(l\left(K_{X / Y}+\Delta\right)+l A\right) \rightarrow \mathcal{O}_{X}\left(l\left(K_{X / Y}+\Delta\right)+l A\right)\right) .
$$

If we take a Zariski open set $U^{\prime}$ of $Y$ such that $\operatorname{codim}_{Y}\left(Y \backslash U^{\prime}\right) \geq 2$ suitably, then $\psi_{*} \mathcal{O}_{X}\left(l\left(K_{X / Y}+\Delta\right)+l A\right)$ is locally free and $\psi_{*} \mathcal{O}_{X}\left(l\left(K_{X / Y}+\right.\right.$ $\Delta)+l A) \simeq \psi_{*} \mathcal{M}$ over $U^{\prime}$ by construction. Therefore, $f_{*} \mathcal{O}_{X}\left(l\left(K_{X / Y}+\right.\right.$ $\Delta)+l A) \simeq f_{*} \mathcal{M}$ on $U^{\prime}$. Since

$$
\widehat{S}^{N}\left(f_{*} \mathcal{O}_{X}\left(l\left(K_{X / Y}+\Delta\right)+l A\right)\right)
$$

is generically generated by global sections, there is an effective divisor $S$ on $X$ such that $\operatorname{codim}_{Y} f(S) \geq 2$ and that $\mathcal{M}^{N} \otimes \mathcal{O}_{X}(N l S)$ is generated by global sections over $f^{-1}(U) \subset X$, where $U$ is a nonempty Zariski open set of $Y$. We put

$$
L=K_{X / Y}+\Delta^{=1}+l\{\Delta\}+A+S
$$

and

We note that

$$
L^{(l-1)}=(l-1) L-\left\lfloor\frac{l-1}{l}(E+(l-1) l\{\Delta\})\right\rfloor .
$$

$$
\Delta^{=1}=\lfloor\Delta\rfloor \text { and } \Delta=\Delta^{=1}+\{\Delta\}
$$


because $\Delta$ is a boundary $\mathbb{Q}$-divisor. We also note that

$$
\begin{aligned}
l L & =l K_{X / Y}+l \Delta+l A+l S+(l-1) l\{\Delta\} \\
& =\mathcal{M}+l S+E+(l-1) l\{\Delta\} .
\end{aligned}
$$

By the usual covering argument, we obtain that

$$
f_{*} \mathcal{O}_{X}\left(K_{X / Y}+\Delta^{=1}+L^{(l-1)}\right)
$$

is weakly positive by Lemma 8.1 . We can easily see that

$$
\begin{aligned}
& K_{X / Y}+\Delta^{=1}+(l-1) L-\left\lfloor(l-1)^{2}\{\Delta\}\right\rfloor \\
& =l\left(K_{X / Y}+\Delta^{=1}\right)+l(l-1)\{\Delta\}-\left\lfloor\left(l^{2}-2 l+1\right)\{\Delta\}\right\rfloor+(l-1)(A+S) \\
& =l\left(K_{X / Y}+\Delta\right)+(l-1)(A+S) .
\end{aligned}
$$

Therefore, we can also check that

$$
\begin{aligned}
& f_{*} \mathcal{O}_{X}\left(K_{X / Y}+\Delta^{=1}+L^{(l-1)}\right) \\
& \subset f_{*} \mathcal{O}_{X}\left(K_{X / Y}+\Delta^{=1}+(l-1) L-\left\lfloor(l-1)^{2}\{\Delta\}\right\rfloor\right)
\end{aligned}
$$

and that they coincide over the generic point of $Y$ because $E$ is the relative base locus of $l\left(K_{X / Y}+\Delta\right)+l A, A=f^{*} A^{\prime}, f(S) \subsetneq Y$, and

$$
L^{(l-1)}=(l-1) L-\left\lfloor(l-1)^{2}\{\Delta\}\right\rfloor-\left\lfloor\frac{l-1}{l} E\right\rfloor .
$$

Hence we obtain that

$$
\begin{aligned}
& f_{*} \mathcal{O}_{X}\left(l\left(K_{X / Y}+\Delta\right)+(l-1)(A+S)\right) \\
& =f_{*} \mathcal{O}_{X}\left(K_{X / Y}+\Delta^{=1}+(l-1) L-\left\lfloor(l-1)^{2}\{\Delta\}\right\rfloor\right)
\end{aligned}
$$

is weakly positive by Lemma 7.7. Therefore,

$$
\left(f_{*} \mathcal{O}_{X}\left(l\left(K_{X / Y}+\Delta\right)+(l-1) A\right)\right)^{* *}
$$

is weakly positive because $\operatorname{codim}_{Y} f(S) \geq 2$. This means that

$$
f_{*} \mathcal{O}_{X}\left(l\left(K_{X / Y}+\Delta\right)+(l-1) A\right)
$$

is weakly positive (see Remark 7.4).

Let us start the proof of Theorem 1.1.

Proof of Theorem 1.1. We divide the proof into several steps.

Step 1. Let $\widetilde{X} \rightarrow X$ be a resolution such that $\widetilde{X}$ is a compact Kähler manifold with

$$
K_{\tilde{X}}+\widetilde{\Delta}=\pi^{*}\left(K_{X}+\Delta\right)+E,
$$

where $E$ and $\widetilde{\Delta}$ are effective and have no common components. By replacing $(X, \Delta)$ with $(\widetilde{X}, \widetilde{\Delta})$, we may assume that $X$ is a compact 
Kähler manifold and that $\operatorname{Supp} \Delta$ is a simple normal crossing divisor. By replacing $m k$ with $k$, we may assume that $m=1$.

Step 2. Let $H$ be an ample Cartier divisor on $Y$. We put $r=\min \left\{s>0 ; f_{*} \mathcal{O}_{X}\left(k\left(K_{X / Y}+\Delta\right)\right) \otimes \mathcal{O}_{Y}((s k-1) H)\right.$ is weakly positive $\}$. By definition, we can find $\nu>0$ such that

$$
\widehat{S}^{\nu}\left(f_{*} \mathcal{O}_{X}\left(k\left(K_{X / Y}+\Delta\right)\right)\right) \otimes \mathcal{O}_{Y}((r k \nu-\nu) H) \otimes \mathcal{O}_{Y}(\nu H)
$$

is generated by global sections over a nonempty Zariski open set. By Lemma 8.2,

$$
f_{*} \mathcal{O}_{X}\left(k\left(K_{X / Y}+\Delta\right) \otimes \mathcal{O}_{Y}((r k-r) H)\right.
$$

is weakly positive. The choice of $r$ allows this only if $(r-1) k-1<r k-r$, equivalently, $r \leq k$. Hence we obtained the weak positivity of

$$
f_{*} \mathcal{O}_{Y}\left(k\left(K_{X / Y}+\Delta\right)\right) \otimes \mathcal{O}_{Y}\left(\left(k^{2}-k\right) H\right) .
$$

Step 3. Let $d$ be an arbitrary positive integer. By Lemma 8.3, we can take a finite flat morphism $g: Y^{\prime} \rightarrow Y$ from a smooth projective variety $Y^{\prime}$ such that $g^{*} H \sim d H^{\prime}$ and that $X^{\prime}=X \times_{Y} Y^{\prime}$ is a compact Kähler manifold. We put $\tau: X^{\prime} \rightarrow X$ and $\Delta^{\prime}=\tau^{*} \Delta$. Thus, by Lemma 8.3 , we have

$$
f_{*}^{\prime} \mathcal{O}_{X^{\prime}}\left(k\left(K_{X^{\prime} / Y^{\prime}}+\Delta^{\prime}\right)\right) \simeq g^{*} f_{*} \mathcal{O}_{X}\left(k\left(K_{X / Y}+\Delta\right)\right),
$$

where $f^{\prime}: X^{\prime} \rightarrow Y^{\prime}$. We may further assume that $\Delta^{\prime}$ is a boundary $\mathbb{Q}$ divisor such that $\operatorname{Supp} \Delta^{\prime}$ is a simple normal crossing divisor by Lemma 8.3. Then we obtain that

$$
g^{*} f_{*} \mathcal{O}_{X}\left(k\left(K_{X / Y}+\Delta\right)\right) \otimes \mathcal{O}_{Y^{\prime}}\left(\left(k^{2}-k\right) H^{\prime}\right)
$$

is weakly positive. This is because

$$
f_{*}^{\prime} \mathcal{O}_{X^{\prime}}\left(k\left(K_{X^{\prime} / Y^{\prime}}+\Delta^{\prime}\right)\right) \otimes \mathcal{O}_{Y^{\prime}}\left(\left(k^{2}-k\right) H^{\prime}\right)
$$

is weakly positive by applying the above result (see Step 2) to $f^{\prime}$ : $\left(X^{\prime}, \Delta^{\prime}\right) \rightarrow Y^{\prime}$. If $\alpha$ is a positive integer, then we choose $d=2 \alpha\left(k^{2}-\right.$ $k)+1$. Let $\beta$ be a sufficiently large positive integer. Then we have that

$$
\begin{aligned}
& \widehat{S}^{2 \alpha \beta}\left(g^{*} f_{*} \mathcal{O}_{X}\left(k\left(K_{X / Y}+\Delta\right)\right) \otimes \mathcal{O}_{Y^{\prime}}\left(\left(k^{2}-k\right) H^{\prime}\right)\right) \otimes \mathcal{O}_{Y^{\prime}}\left(\beta H^{\prime}\right) \\
& =g^{*} \widehat{S}^{2 \alpha \beta}\left(f_{*} \mathcal{O}_{X}\left(k\left(K_{X / Y}+\Delta\right)\right)\right) \otimes g^{*} \mathcal{O}_{Y}(\beta H)
\end{aligned}
$$

is generated by global sections over a nonempty Zariski open set. We may further assume that $g_{*} \mathcal{O}_{Y^{\prime}} \otimes \mathcal{O}_{Y}(\beta H)$ is generated by global sections. Over the Zariski open set $\widehat{Y}$ of $Y$ where

$$
\widehat{S}^{2 \alpha \beta}\left(f_{*} \mathcal{O}_{X}\left(k\left(K_{X / Y}+\Delta\right)\right)\right)
$$


is locally free, we have a surjection

$$
\begin{gathered}
g_{*} g^{*} \widehat{S}^{2 \alpha \beta}\left(f_{*} \mathcal{O}_{X}\left(k\left(K_{X / Y}+\Delta\right)\right)\right) \otimes \mathcal{O}_{Y}(\beta H) \\
\rightarrow \widehat{S}^{2 \alpha \beta}\left(f_{*} \mathcal{O}_{X}\left(k\left(K_{X / Y}+\Delta\right)\right)\right) \otimes \mathcal{O}_{Y}(\beta H) .
\end{gathered}
$$

Therefore, we have a homomorphism

$$
\bigoplus_{\text {finite }}\left(\mathcal{O}_{Y}(\beta H) \otimes g_{*} \mathcal{O}_{Y^{\prime}}\right) \rightarrow \widehat{S}^{2 \alpha \beta}\left(f_{*} \mathcal{O}_{X}\left(k\left(K_{X / Y}+\Delta\right)\right)\right) \otimes \mathcal{O}_{Y}(2 \beta H)
$$

which is surjective over a nonempty Zariski open set. Note that $g_{*} \mathcal{O}_{Y^{\prime}} \otimes$ $\mathcal{O}_{Y}(\beta H)$ is generated by global sections. Therefore,

$$
\widehat{S}^{2 \alpha \beta}\left(f_{*} \mathcal{O}_{X}\left(k\left(K_{X / Y}+\Delta\right)\right)\right) \otimes \mathcal{O}_{Y}(2 \beta H)
$$

is generated by global sections over a nonempty Zariski open set.

This means that $f_{*} \mathcal{O}_{X}\left(k\left(K_{X / Y}+\Delta\right)\right)$ is weakly positive.

In the above proof of Theorem 1.1, we have already used the following lemma. It is a variant of Kawamata's cover (see [Kw1, Theorem 17]). The description of Kawamata's covering trick in [EV, 3.19. Lemma] is very useful for our purpose. See also [AK, 5.3. Kawamata's covering] and [V4, Lemma 2.5].

Lemma 8.3. Let $f: X \rightarrow Y$ be a surjective morphism from a compact Kähler manifold $X$ to a smooth projective variety $Y$ and let $H$ be a Cartier divisor on $Y$. Let $d$ be an arbitrary positive integer. Then we can take a finite flat morphism $g: Y^{\prime} \rightarrow Y$ from a smooth projective variety $Y^{\prime}$ and a Cartier divisor $H^{\prime}$ on $Y^{\prime}$ such that $g^{*} H \sim d H^{\prime}$ and that $X^{\prime}=X \times_{Y} Y^{\prime}$ is a compact Kähler manifold with $\omega_{X^{\prime} / Y^{\prime}}=\tau^{*} \omega_{X / Y}$, where $\tau: X^{\prime} \rightarrow X$. Let $S$ be a simple normal crossing divisor on $X$. Then we can choose $g: Y^{\prime} \rightarrow Y$ such that $\tau^{*} S$ is a simple normal crossing divisor on $X^{\prime}$.

Furthermore, let $D$ be a Cartier divisor on $X$. We put $f^{\prime}: X^{\prime} \rightarrow Y^{\prime}$. Then there is a natural isomorphism

$$
f_{*}^{\prime} \mathcal{O}_{X^{\prime}}\left(n K_{X^{\prime} / Y^{\prime}}+\tau^{*} D\right) \simeq g^{*} f_{*} \mathcal{O}_{X}\left(n K_{X / Y}+D\right)
$$

for every integer $n$.

Proof. We take general very ample Cartier divisors $D_{1}$ and $D_{2}$ with the following properties.

(i) $H \sim D_{1}-D_{2}$,

(ii) $D_{1}, D_{2}, f^{*} D_{1}$, and $f^{*} D_{2}$ are smooth,

(iii) $D_{1}$ and $D_{2}$ have no common components, and

(iv) $\operatorname{Supp}\left(D_{1}+D_{2}\right)$ and $\operatorname{Supp}\left(f^{*} D_{1}+f^{*} D_{2}\right)$ are simple normal crossing divisors. 
We take a finite flat cover due to Kawamata with respect to $Y$ and $D_{1}+D_{2}$ (see [Kw1, Theorem 17]), we obtain $g: Y^{\prime} \rightarrow Y$ and $H^{\prime}$ such that $g^{*} H \sim d H^{\prime}$. By the construction of the above Kawamata cover $g: Y^{\prime} \rightarrow Y$, we may assume that the ramification locus $\Sigma$ of $g$ in $Y$ is a general simple normal crossing divisor. This means that $f^{*} P$ is a smooth divisor for any irreducible component $P$ of $\Sigma$ and that $f^{*} \Sigma$ is a simple normal crossing divisor on $X$. We may further assume that $f^{*} P \not \subset S$ for any irreducible component $P$ of $\Sigma$ and that $f^{*} \Sigma+S$ is a simple normal crossing divisor on $X$ since $D_{1}$ and $D_{2}$ are general. In this situation, we can check that $X^{\prime}=X \times_{Y} Y^{\prime}$ is a compact Kähler manifold (see Remark 2.12) and that $\tau^{*} S$ is a simple normal crossing divisor on $X^{\prime}$.

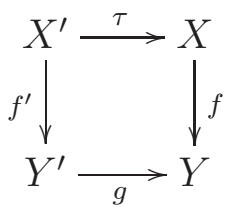

By construction, we can also easily check that $\omega_{X^{\prime} / Y^{\prime}}=\tau^{*} \omega_{X / Y}$ by the Hurwitz formula. Therefore, we have

$$
\mathcal{O}_{X^{\prime}}\left(n K_{X^{\prime} / Y^{\prime}}+\tau^{*} D\right) \simeq \tau^{*} \mathcal{O}_{X}\left(n K_{X / Y}+D\right)
$$

for every integer $n$. Thus, we obtain

$$
\begin{aligned}
f_{*}^{\prime} \mathcal{O}_{X^{\prime}}\left(n K_{X^{\prime} / Y^{\prime}}+\tau^{*} D\right) & \simeq f_{*}^{\prime} \tau^{*} \mathcal{O}_{X}\left(n K_{X / Y}+D\right) \\
& \simeq g^{*} f_{*} \mathcal{O}_{X}\left(n K_{X / Y}+D\right)
\end{aligned}
$$

for every integer $n$.

As a special case of Theorem 1.1, we have:

Corollary 8.4 (cf. [V2, Theorem III]). Let $f: X \rightarrow Y$ be a surjective morphism such that $X$ is a complex manifold in Fujiki's class $\mathcal{C}$ and $Y$ is a smooth projective variety. Then $f_{*} \mathcal{O}_{X}\left(k K_{X / Y}\right)$ is weakly positive for every $k>0$.

As is well-known, Corollary 8.4 is a very famous fundamental result by Viehweg when $X$ is projective.

Remark 8.5. In Corollary 8.4, we did not check the weak positivity of $f_{*} \mathcal{O}_{X}\left(k K_{X / Y}\right)$ in the sense of Definition 7.3. Corollary 8.4 says that $f_{*} \mathcal{O}_{X}\left(k K_{X / Y}\right)$ is weakly positive in the sense of Definition 7.2.

By Theorem 1.1, we can recover Campana's twisted weak positivity (see [Ca, Theorem 4.13]). 
Corollary 8.6. Let $f: X \rightarrow Y$ be a surjective morphism from a complex manifold $X$ in Fujiki's class $\mathcal{C}$ to a smooth projective variety $Y$. Let $D$ be a divisor on $X$. We put $D=D^{h}+D^{v}$ where $D^{h}\left(\right.$ resp. $\left.D^{v}\right)$ is the horizontal (resp. vertical) part of $D$ with respect to $f: X \rightarrow$ $Y$. Assume that Supp $D^{h}$ is a simple normal crossing divisor and the coefficients of $D^{h}$ is less than or equal to $m$, where $m$ is a positive integer. Then $f_{*} \mathcal{O}_{X}\left(m K_{X / Y}+D\right)$ is weakly positive.

Proof. We put $\Delta=\frac{1}{m} D$. Then $(X, \Delta)$ is $\log$ canonical over the generic point of $Y$. We take a resolution $g: X^{\prime} \rightarrow X$. Then

$$
K_{X^{\prime}}+\Delta^{\prime}=g^{*}\left(K_{X}+\Delta\right)+E
$$

where $\Delta^{\prime}$ and $E$ are effective and have no common components such that $\operatorname{Supp}\left(\Delta^{\prime}+E\right)$ is a simple normal crossing divisor on $X^{\prime}$. Let $\widetilde{\Delta}$ be the horizontal part of $\Delta^{\prime}$. Then $\left(X^{\prime}, \widetilde{\Delta}\right)$ is $\log$ canonical. By Lemma 7.7, we can replace $(X, \Delta)$ with $\left(X^{\prime}, \widetilde{\Delta}\right)$. By Theorem 1.1, we obtain that $f_{*} \mathcal{O}_{X}\left(m\left(K_{X / Y}+\Delta\right)\right)=f_{*} \mathcal{O}_{X}\left(m K_{X / Y}+D\right)$ is weakly positive.

\section{AdDition FORMula}

Theorem 1.7 is an easy application of Theorem 1.1. It is contained in [Ca]. See also [L] and [N2].

Proposition 9.1 is a slight reformulation of [V2, Corollary 7.1].

Proposition 9.1 (cf. [V2, Corollary 7.1]). Let $f: V \rightarrow W$ be an equidimensional surjective morphism from a normal projective variety $V$ to a smooth projective variety $W$ with connected fibers. Let $(V, \Delta)$ be a log canonical pair. Let $H$ be an ample Cartier divisor on $W$. Then there are some positive integers $a$ and $l$ such that $a\left(K_{V}+\Delta\right)$ is Cartier and the linear system $\Lambda$ associated to

$$
H^{0}\left(V, \mathcal{O}_{V}\left(a l\left(K_{V / W}+\Delta\right)\right) \otimes f^{*} \mathcal{O}_{W}(l H)\right)
$$

defines a rational map $\Phi: V \rightarrow X$ with

$$
\operatorname{dim} X=\kappa\left(V_{w}, K_{V_{w}}+\left.\Delta\right|_{V_{w}}\right)+\operatorname{dim} W,
$$

where $V_{w}$ is a sufficiently general fiber of $f$. Moreover, there is a rational map $\pi: X \rightarrow W$ such that $f=\pi \circ \Phi$.

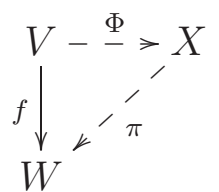

Remark 9.2. When $\kappa\left(V_{w}, K_{V_{w}}+\left.\Delta\right|_{V_{w}}\right)=-\infty$, we claim nothing in Proposition 9.1. 
Proof. We take a positive integer a such that $a\left(K_{V}+\Delta\right)$ is Cartier and $f_{*} \mathcal{O}_{V}\left(a\left(K_{V / W}+\Delta\right)\right)$ is nontrivial. By the twisted weak positivity theorem: Theorem 1.1, there is some $b>0$ such that

$$
\widehat{S}^{2 b}\left(f_{*} \mathcal{O}_{V}\left(a\left(K_{V / W}+\Delta\right)\right)\right) \otimes \mathcal{O}_{W}(b H)
$$

is generated by global sections on some nonempty Zariski open set. Since the natural map

$$
\begin{aligned}
& \widehat{S}^{2 b}\left(f_{*} \mathcal{O}_{V}\left(a\left(K_{V / W}+\Delta\right)\right)\right) \otimes \mathcal{O}_{W}(b H) \\
& \rightarrow f_{*} \mathcal{O}_{V}\left(2 b a\left(K_{V / W}+\Delta\right)\right) \otimes \mathcal{O}_{W}(b H)
\end{aligned}
$$

is nontrivial, we obtain an inclusion from $f^{*} \mathcal{O}_{W}(b H)$ into $\mathcal{O}_{V}\left(2 b a\left(K_{V / W}+\right.\right.$ $\Delta)) \otimes f^{*} \mathcal{O}_{W}(2 b H)$. Note that $f_{*} \mathcal{O}_{V}\left(2 b a\left(K_{V / W}+\Delta\right)\right)$ is reflexive since $f$ is equidimensional. Without loss of generality, we may assume that $b H$ is very ample by replacing $b$ with $b^{\prime} b$ for some $b^{\prime} \gg 0$. We put $l=2 b$. If $b$ is sufficiently large, then the sections of $\mathcal{O}_{V}\left(2 b a\left(K_{V / W}+\right.\right.$ $\Delta)) \otimes f^{*} \mathcal{O}_{W}(2 b H)$ define a rational map $\Phi: V \rightarrow X$ such that $\mathbb{C}(X)$ is algebraically closed in $\mathbb{C}(V)$. Since $b H$ is very ample and there is an inclusion from $f^{*} \mathcal{O}_{W}(b H)$ into $\mathcal{O}_{V}\left(2 b a\left(K_{V / W}+\Delta\right)\right) \otimes f^{*} \mathcal{O}_{W}(2 b H)$, we obtain a rational map $\pi: X \rightarrow W$ such that $f=\pi \circ \Phi$. The easy addition formula gives

$$
\begin{aligned}
& \kappa\left(V_{w}, K_{V_{w}}+\left.\Delta\right|_{V_{w}}\right) \\
& \leq \operatorname{dim} \Phi\left(V_{w}\right)+\kappa\left(F,\left.\left(\mathcal{O}_{V}\left(2 b a\left(K_{V / W}+\Delta\right)\right) \otimes f^{*} \mathcal{O}_{W}(2 b H)\right)\right|_{F}\right) \\
& =\operatorname{dim} \Phi\left(V_{w}\right)
\end{aligned}
$$

where $F$ is a sufficiently general fiber of $\Phi: V \rightarrow X$ (if necessary, we take an elimination of points of indeterminacy of $\Phi)$. On the other hand, the restriction of the linear system $\Lambda$ to $V_{w}$ is a subsystem of $H^{0}\left(V_{w}, \mathcal{O}_{V_{w}}\left(2 b a\left(K_{V_{w}}+\left.\Delta\right|_{V_{w}}\right)\right)\right.$. Therefore, $\operatorname{dim} \Phi\left(V_{w}\right) \leq \kappa\left(V_{w}, K_{V_{w}}+\right.$ $\left.\left.\Delta\right|_{V_{w}}\right)$. Hence, we obtain

$$
\kappa\left(V_{w}, K_{V_{w}}+\left.\Delta\right|_{V_{w}}\right)=\operatorname{dim} \Phi\left(V_{w}\right)=\operatorname{dim} X-\operatorname{dim} W .
$$

Remark 9.3. In Proposition 9.1, it is sufficient to assume that $a$ is a positive integer such that $a\left(K_{V}+\Delta\right)$ is Cartier and that $f_{*} \mathcal{O}_{V}\left(a\left(K_{V / W}+\right.\right.$ $\Delta)$ ) is nontrivial.

Let us start the proof of Theorem 1.7. 
Proof of Theorem 1.\%. By [AK], we can construct a commutative diagram:

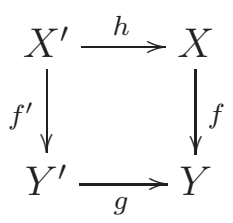

with the following properties:

(i) $f^{\prime}: X^{\prime} \rightarrow Y^{\prime}$ is an equidimensional surjective morphism from a normal projective variety $X^{\prime}$ to a smooth projective variety $Y^{\prime}$,

(ii) $h$ and $g$ are birational, and

(iii) $X^{\prime}$ has only quotient singularities and $\left(U_{X^{\prime}} \subset X^{\prime}\right)$ is toroidal for some nonempty Zariski open set $U_{X^{\prime}}$.

By $[\mathrm{AK}]$, we may assume that $\operatorname{Exc}(h) \cup \operatorname{Supp}_{*}^{-1} \Delta$ is contained in $X^{\prime} \backslash U_{X^{\prime}}$. We put

$$
K_{X^{\prime}}+\Delta^{\prime}=h^{*}\left(K_{X}+\Delta\right)+E
$$

such that $\left(X^{\prime}, \Delta^{\prime}\right)$ is log canonical and $E$ is effective and $h$-exceptional. Let $H$ be an ample Cartier divisor on $Y^{\prime}$. Since $K_{Y^{\prime}}$ is a big divisor, by Kodaira's lemma, $a K_{Y^{\prime}} \sim H+F$ for some effective divisor $F$ on $Y^{\prime}$ and a sufficiently divisible positive integer $a$. By Proposition 9.1 (see also Remark 9.3), we have

$$
\begin{aligned}
\kappa\left(X, K_{X}+\Delta\right) & =\kappa\left(X^{\prime}, K_{X^{\prime}}+\Delta^{\prime}\right) \\
& \geq \kappa\left(X^{\prime}, a\left(K_{X^{\prime}}+\Delta^{\prime}\right)-a f^{\prime *} K_{Y^{\prime}}+f^{\prime *} H\right) \\
& \geq \kappa\left(X_{y}^{\prime}, K_{X_{y}^{\prime}}+\left.\Delta^{\prime}\right|_{X_{y}^{\prime}}\right)+\operatorname{dim} Y^{\prime} \\
& =\kappa\left(X_{y}, K_{X_{y}}+\left.\Delta\right|_{X_{y}}\right)+\operatorname{dim} Y .
\end{aligned}
$$

Note that

$$
\kappa\left(X, K_{X}+\Delta\right) \leq \kappa\left(X_{y}, K_{X_{y}}+\left.\Delta\right|_{X_{y}}\right)+\operatorname{dim} Y
$$

always holds by the easy addition formula. Therefore, we obtain

$$
\kappa\left(X, K_{X}+\Delta\right)=\kappa\left(X_{y}, K_{X_{y}}+\left.\Delta\right|_{X_{y}}\right)+\operatorname{dim} Y .
$$

This is the desired equality.

\section{AdDition FOR LOGARITHMiC KodAirA Dimensions}

We prove Theorem 1.9, which is due to Maehara (see [Ma]), as an application of Theorem 1.1: Twisted weak positivity. Before we start the proof of Theorem 1.9, we give some comments on Maehara's works (see $[\mathrm{Ma}])$. 
Remark 10.1. In Theorem 1.9,

$$
f_{*} \mathcal{O}_{X}\left(k\left(K_{X}+D_{X}\right)\right) \otimes \mathcal{O}_{Y}\left(-k\left(K_{Y}+D_{Y}\right)\right) \otimes \mathcal{O}_{Y}\left(D_{Y}\right)
$$

is weakly 1-positive in the sense of Maehara for any $k>0$. It is the main theorem of [Ma]. Maehara obtained Theorem 1.9 as a corollary of the above weak 1-positivity (see [Ma, Corollary 2]). In this section, we do not use Maehara's results and prove Theorem 1.9 as an application of Theorem 1.1 by using the weak semistable reduction theorem (see $[\mathrm{AK}])$.

Let us start the proof of Theorem 1.9.

Proof of Theorem 1.9. By [AK], we may assume that

(i) $f:\left(U_{X} \subset X\right) \rightarrow\left(U_{Y} \subset Y\right)$ is toroidal and is equidimensional,

(ii) $D_{Y}$ is contained in $Y \backslash U_{Y}$ and $D_{X}$ is contained in $X \backslash U_{X}$,

(iii) $X$ has only quotient singularities, $Y$ is smooth, and

(iv) $f$ is smooth over $U_{Y}$.

Moreover, there is a Kawamata cover $\tau: Y^{\prime} \rightarrow Y$ such that the normalization $X^{\prime}$ of $\widetilde{X}=X \times_{Y} Y^{\prime}$ is a weak semistable reduction over $Y^{\prime}$. Note that $f$ is flat. We put $\Delta_{Y}=Y \backslash U_{Y}$.

Lemma 10.2 (cf. [Ma, Main Theorem]). Under the above assumptions,

$$
f_{*} \mathcal{O}_{X}\left(k\left(K_{X}+D_{X}\right)\right) \otimes \mathcal{O}_{Y}\left(-k\left(K_{Y}+D_{Y}\right)\right) \otimes \mathcal{O}_{Y}\left(\Delta_{Y}\right)
$$

is weakly positive for every divisible positive integer $k$.

Once we establish Lemma 10.2, we can check:

Lemma 10.3. Let $H$ be an ample Cartier divisor on $Y$. Then there are some positive integers a and $l$ such that $a\left(K_{X}+D_{X}\right)$ is Cartier and the linear system $\Lambda$ associated to

$$
H^{0}\left(X, \mathcal{O}_{X}\left(\operatorname{al}\left(K_{X / Y}+D_{X}-f^{*} D_{Y}\right)\right) \otimes f^{*} \mathcal{O}_{Y}\left(l H+l \Delta_{Y}\right)\right)
$$

defines a rational map $\Phi: X \rightarrow Z$ with

$$
\operatorname{dim} Z=\kappa\left(F, K_{F}+\left.D_{X}\right|_{F}\right)+\operatorname{dim} Y
$$

where $F$ is a sufficiently general fiber of $f$. Moreover, there is a rational map $\pi: Z \rightarrow Y$ such that $f=\pi \circ \Phi$.

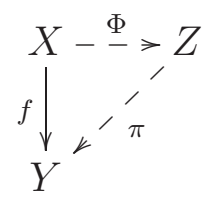

Proof. See the proof of Proposition 9.1 (see also Remark 9.3). 
If $a$ is a sufficiently large and divisible positive integer, then $a\left(K_{X}+\right.$ $\left.D_{X}\right)$ is Cartier,

$$
f_{*} \mathcal{O}_{X}\left(a\left(K_{X}+D_{X}\right)\right) \otimes \mathcal{O}_{Y}\left(-a\left(K_{Y}+D_{Y}\right)\right) \otimes \mathcal{O}_{Y}\left(\Delta_{Y}\right) \neq 0
$$

is weakly positive by Lemma 10.2 , and

$$
a\left(K_{Y}+D_{Y}\right)-\Delta_{Y} \sim H+G
$$

for an ample Cartier divisor $H$ and some effective divisor $G$ on $Y$ by Kodaira's lemma. Therefore, by Lemma 10.3, we obtain

$$
\begin{aligned}
& \kappa\left(X, K_{X}+D_{X}\right) \\
& \geq \kappa\left(X, a\left(K_{X}+D_{X}\right)-a f^{*}\left(K_{Y}+D_{Y}\right)+f^{*} \Delta_{Y}+f^{*} H\right) \\
& \geq \kappa\left(F, K_{F}+\left.D_{X}\right|_{F}\right)+\operatorname{dim} Y .
\end{aligned}
$$

On the other hand, we always have

$$
\kappa\left(X, K_{X}+D_{X}\right) \leq \kappa\left(F, K_{F}+\left.D_{X}\right|_{F}\right)+\operatorname{dim} Y
$$

by the easy addition formula. Thus, we obtain

$$
\kappa\left(X, K_{X}+D_{X}\right)=\kappa\left(F, K_{F}+\left.D_{X}\right|_{F}\right)+\operatorname{dim} Y .
$$

This is the desired equality.

Anyway, we see that it is sufficient to prove Lemma 10.2. For the proof of Lemma 10.2, we can replace $D_{Y}$ with $\Delta_{Y}$ and $D_{X}$ with $\operatorname{Supp}\left(D_{X}+f^{*} D_{Y}\right)$. Moreover, by adding some divisors to $D_{Y}$, we may further assume that $D_{Y}$ is a simple normal crossing divisor, $\Delta_{Y} \leq D_{Y}$, and $\tau$ is étale over $Y \backslash D_{Y}$. We put $f^{\prime}: X^{\prime} \rightarrow Y^{\prime}, p: X^{\prime} \rightarrow \widetilde{X}$, $q: \widetilde{X} \rightarrow X$, and $K_{X^{\prime}}+D_{X^{\prime}}=\lambda^{*}\left(K_{X}+D_{X}\right)$ where $\lambda=q \circ p: X^{\prime} \rightarrow X$.

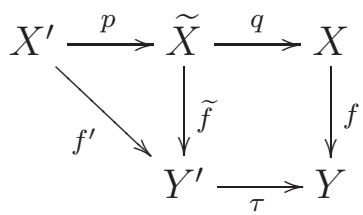

We also put $K_{Y^{\prime}}+D_{Y^{\prime}}=\tau^{*}\left(K_{Y}+D_{Y}\right)$. Note that $D_{X^{\prime}}$ and $D_{Y^{\prime}}$ are reduced. Let $\operatorname{Sing}(X)$ be the singular locus of $X$. We put $\Sigma=$ $f^{-1}(f(\operatorname{Sing}(X)))$ and $\widetilde{\Sigma}=q^{-1}(\Sigma)$. Then $\operatorname{codim}_{X} \Sigma \geq 2, \operatorname{codim}_{\widetilde{X}} \widetilde{\Sigma} \geq 2$ and $\left.\omega_{\tilde{X}}\right|_{X^{\dagger}}$ is locally free by the flat base change theorem (cf. Theorem 2.16) with $X^{\dagger}=\widetilde{X} \backslash \widetilde{\Sigma}$. We put $\omega_{\widetilde{X}}^{[k]}=\iota_{*}\left(\left(\left.\omega_{\widetilde{X}}\right|_{X^{\dagger}}\right)^{\otimes k}\right)$ where $\iota: X^{\dagger} \rightarrow$ $\widetilde{X}$. We note that $\widetilde{X}$ is Cohen-Macaulay by the local description of Kawamata's cover. We also note that $\omega_{\widetilde{X}}^{[k]}$ is invertible if $\mathcal{O}_{X}\left(k K_{X}\right)$ is invertible. We can check: 
Lemma 10.4 (cf. [Ma, Lemma C]). We have the following inclusion

$$
\begin{aligned}
(\boldsymbol{A}) \quad & p_{*} \mathcal{O}_{X^{\prime}}\left(k\left(K_{X^{\prime} / Y^{\prime}}+D_{X^{\prime}}-f^{\prime *} D_{Y^{\prime}}\right)\right) \\
& \subset \omega_{\widetilde{X} / Y^{\prime}}^{[k]} \otimes \mathcal{O}_{\widetilde{X}}\left(k\left(q^{*}\left(D_{X}-f^{*} D_{Y}\right)\right)\right) \otimes \mathcal{O}_{\widetilde{X}}\left(q^{*} f^{*} \Delta_{Y}\right)
\end{aligned}
$$

for every divisible positive integer $k$.

Proof of Lemma 10.4. Since $k$ is divisible, we have that $\omega_{\widetilde{X} / Y^{\prime}}^{[k]}$ is invertible and that $k\left(K_{X^{\prime} / Y^{\prime}}+D_{X^{\prime}}-f^{\prime *} D_{Y^{\prime}}\right)$ and $k\left(q^{*}\left(D_{X}-f^{*} D_{Y}\right)\right)$ are Cartier. Therefore, the both hand sides satisfy Serre's $S_{2}$ condition. We also note that $X \backslash \Sigma$ is smooth and that $X^{\dagger}$ is Gorenstein. By replacing $X$ and $\tilde{X}$ with $X \backslash \Sigma$ and $X^{\dagger}$ respectively, we may assume that $X$ is smooth and that $\widetilde{X}$ is Gorenstein. We have

$$
p_{*} \mathcal{O}_{X^{\prime}}\left(K_{X^{\prime}}\right) \subset \omega_{\widetilde{X}}
$$

because $p$ is the normalization. Since $D_{X^{\prime}}-\lambda^{*} D_{X} \leq 0$, we obtain

$$
p_{*} \mathcal{O}_{X^{\prime}}\left(K_{X^{\prime} / Y^{\prime}}+D_{X^{\prime}}-\lambda^{*} D_{X}\right) \subset \omega_{\tilde{X} / Y^{\prime}}
$$

This is equivalent to

$$
\begin{aligned}
& p_{*} \mathcal{O}_{X^{\prime}}\left(K_{X^{\prime} / Y^{\prime}}+D_{X^{\prime}}-f^{\prime *} D_{Y^{\prime}}\right) \otimes \mathcal{O}_{\widetilde{X}}\left(\tilde{f}^{*} D_{Y^{\prime}}\right) \\
& \subset \omega_{\tilde{X} / Y^{\prime}} \otimes \mathcal{O}_{\widetilde{X}}\left(q^{*}\left(D_{X}-f^{*} D_{Y}\right)\right) \otimes \mathcal{O}_{\widetilde{X}}\left(q^{*} f^{*} D_{Y}\right) .
\end{aligned}
$$

Since $\tilde{f}^{*} D_{Y^{\prime}}$ is effective, we have

$$
\begin{aligned}
& p_{*} \mathcal{O}_{X^{\prime}}\left(K_{X^{\prime} / Y^{\prime}}+D_{X^{\prime}}-f^{\prime *} D_{Y^{\prime}}\right) \\
& \subset \omega_{\tilde{X} / Y^{\prime}} \otimes \mathcal{O}_{\tilde{X}}\left(q^{*}\left(D_{X}-f^{*} D_{Y}\right)\right) \otimes \mathcal{O}_{\widetilde{X}}\left(q^{*} f^{*} D_{Y}\right) .
\end{aligned}
$$

Over the generic point of every irreducible component of $D_{Y}-\Delta_{Y}$, $f$ is smooth, $\widetilde{f}$ is smooth, $p$ is an isomorphism, and $D_{X}-f^{*} D_{Y}=$ $D_{X^{\prime}}-f^{\prime *} D_{Y^{\prime}}=0$. Therefore, we obtain

$$
\begin{aligned}
& p_{*} \mathcal{O}_{X^{\prime}}\left(K_{X^{\prime} / Y^{\prime}}+D_{X^{\prime}}-f^{\prime *} D_{Y^{\prime}}\right) \\
& \subset \omega_{\tilde{X} / Y^{\prime}} \otimes \mathcal{O}_{\widetilde{X}}\left(q^{*}\left(D_{X}-f^{*} D_{Y}\right)\right) \otimes \mathcal{O}_{\widetilde{X}}\left(q^{*} f^{*} \Delta_{Y}\right) .
\end{aligned}
$$

We note that

$$
q^{*} \mathcal{O}_{X}\left(K_{X}+D_{X}-f^{*}\left(K_{Y}+D_{Y}\right)\right) \simeq \omega_{\widetilde{X} / Y^{\prime}} \otimes \mathcal{O}_{\widetilde{X}}\left(q^{*}\left(D_{X}-f^{*} D_{Y}\right)\right)
$$

by the flat base change theorem (cf. Theorem 2.16) and

$$
\begin{aligned}
& p^{*} q^{*} \mathcal{O}_{X}\left(K_{X}+D_{X}-f^{*}\left(K_{Y}+D_{Y}\right)\right) \\
& \simeq \mathcal{O}_{X^{\prime}}\left(K_{X^{\prime}}+D_{X^{\prime}}-f^{\prime *}\left(K_{Y^{\prime}}+D_{Y^{\prime}}\right)\right) .
\end{aligned}
$$

By taking

$$
\otimes \omega_{\widetilde{X} / Y^{\prime}}^{\otimes k-1} \otimes \mathcal{O}_{\widetilde{X}}\left((k-1) q^{*}\left(D_{X}-f^{*} D_{Y}\right)\right)
$$

with $(\varnothing)$, we obtain the desired inclusion by the projection formula. 
Let us go back to the proof of Lemma 10.2. Note that

$$
\begin{aligned}
& \omega_{\widetilde{X} / Y^{\prime}}^{[k]} \otimes \mathcal{O}_{\widetilde{X}}\left(k\left(q^{*}\left(D_{X}-f^{*} D_{Y}\right)\right)\right) \\
& \simeq q^{*}\left(\mathcal{O}_{X}\left(k\left(K_{X}+D_{X}\right)\right) \otimes f^{*} \mathcal{O}_{Y}\left(-k\left(K_{Y}+D_{Y}\right)\right)\right)
\end{aligned}
$$

when $k$ is a divisible positive integer. By applying $\widetilde{f}_{*},(\boldsymbol{\Lambda})$ implies:

Lemma 10.5. There exists a generically isomorphic inclusion

$$
\begin{aligned}
& f_{*}^{\prime} \mathcal{O}_{X^{\prime}}\left(k\left(K_{X^{\prime}}+D_{X^{\prime}}\right)\right) \otimes \mathcal{O}_{Y^{\prime}}\left(-k\left(K_{Y^{\prime}}+D_{Y^{\prime}}\right)\right) \\
& \subset \tau^{*}\left(f_{*} \mathcal{O}_{X}\left(k\left(K_{X}+D_{X}\right)\right) \otimes \mathcal{O}_{Y}\left(-k\left(K_{Y}+D_{Y}\right)\right) \otimes \mathcal{O}_{Y}\left(\Delta_{Y}\right)\right)
\end{aligned}
$$

for every divisible positive integer $k$.

This is because $g$ is flat. Since

$$
\begin{aligned}
& f_{*}^{\prime} \mathcal{O}_{X^{\prime}}\left(k\left(K_{X^{\prime}}+D_{X^{\prime}}\right)\right) \otimes \mathcal{O}_{Y^{\prime}}\left(-k\left(K_{Y^{\prime}}+D_{Y^{\prime}}\right)\right) \\
& =f_{*}^{\prime} \mathcal{O}_{X^{\prime}}\left(k\left(K_{X^{\prime} / Y^{\prime}}+D_{X^{\prime}}-f^{\prime *} D_{Y^{\prime}}\right)\right)
\end{aligned}
$$

is weakly positive by Theorem 1.1 , we can easily check that

$$
f_{*} \mathcal{O}_{X}\left(k\left(K_{X}+D_{X}\right)\right) \otimes \mathcal{O}_{Y}\left(-k\left(K_{Y}+D_{Y}\right)\right) \otimes \mathcal{O}_{Y}\left(\Delta_{Y}\right)
$$

is also weakly positive (see, for example, [V2, Lemma 1.4. 5)] and [F15, Lemma 3.6]). Note that $D_{X^{\prime}}-f^{\prime *} D_{Y^{\prime}}$ is effective since $f^{\prime}$ is weakly semistable. Thus we obtain Lemma 10.2. This implies that the equality in Theorem 1.9 holds.

Note that Theorem 1.9 contains a generalization of [Kw1, Theorem 30], which plays an important role for Kawamata's theorem on the quasi-Albanese maps for varieties of the logarithmic Kodaira dimension zero (see [Kw1, Corollary 29]). For the details, see [F14].

\section{REFERENCES}

[AK] D. Abramovich, K. Karu, Weak semistable reduction in characteristic 0, Invent. Math. 139 (2000), no. 2, 241-273.

[A] F. Ambro, An injectivity theorem, Compos. Math. 150 (2014), no. 6, 9991023.

[BS] C. Bănică, O. Stănăşilă, Algebraic methods in the global theory of complex spaces, Translated from the Romanian. Editura Academiei, Bucharest; John Wiley \& Sons, London-New York-Sydney, 1976.

[Ca] F. Campana, Orbifolds, special varieties and classification theory, Ann. Inst. Fourier (Grenoble) 54 (2004), no. 3, 499-630.

[CHP] F. Campana, A. Höring, T. Peternell, Abundance for Kähler theeefolds, preprint (2014). arXiv:1403.3175 [math.AG]

[Co] B. Conrad, Grothendieck duality and base change, Lecture Notes in Mathematics, 1750. Springer-Verlag, Berlin, 2000.

[D] P. Deligne, Théorie de Hodge. III, Inst. Hautes Études Sci. Publ. Math. No. 44 (1974), 5-77. 
[E1] F. El Zein, Mixed Hodge structures, Trans. Amer. Math. Soc. 275 (1983), no. $1,71-106$.

[E2] F. El Zein, Introduction á la théorie de Hodge mixte, Actualités Mathématiques, Hermann, Paris, 1991.

[EL] F. El Zein, D. T. Lê, Mixed Hodge structures, in Hodge theory, edited by E. Cattani, F. El Zein, P. A. Griffiths, D. T. Lê, Mathematical Notes, 49, Princeton University Press, Princeton, NJ, 2014.

[EV] H. Esnault, E. Viehweg, Lectures on vanishing theorems, DMV Seminar, 20. Birkhäuser Verlag, Basel, 1992.

[Fi] G. Fischer, Complex analytic geometry, Lecture Notes in Mathematics, Vol. 538. Springer-Verlag, Berlin-New York, 1976.

[Fk1] A. Fujiki, Duality of mixed Hodge structures of algebraic varieties, Publ. Res. Inst. Math. Sci. 16 (1980), no. 3, 635-667.

[Fk2] A. Fujiki, On the Douady space of a compact complex space in the category $\mathcal{C}$, Nagoya Math. J. 85 (1982), 189-211.

[F1] O. Fujino, Higher direct images of log canonical divisors, J. Differential Geom. 66 (2004), no. 3, 453-479.

[F2] O. Fujino, What is log terminal?, Flips for 3-folds and 4-folds, 49-62, Oxford Lecture Ser. Math. Appl., 35, Oxford Univ. Press, Oxford, 2007.

[F3] O. Fujino, Introduction to the log minimal model program for log canonical pairs, preprint (2008).

[F4] O. Fujino, On injectivity, vanishing and torsion-free theorems for algebraic varieties, Proc. Japan Acad. Ser. A Math. Sci. 85 (2009), no. 8, 95-100.

[F5] O. Fujino, On Kawamata's theorem, Classification of algebraic varieties, 305-315, EMS Ser. Congr. Rep., Eur. Math. Soc., Zürich, 2011.

[F6] O. Fujino, Fundamental theorems for the log minimal model program, Publ. Res. Inst. Math. Sci. 47 (2011), no. 3, 727-789.

[F7] O. Fujino, A transcendental approach to Kollár's injectivity theorem II, J. Reine Angew. Math. 681 (2013), 149-174.

[F8] O. Fujino, Vanishing theorems, to appear in Adv. Stud. Pure Math. arXiv:1202.4200 [math.AG]

[F9] O. Fujino, Semipositivity theorems for moduli problems, preprint (2012). arXiv:1210.5784 [math.AG]

[F10] O. Fujino, Injectivity theorems, to appear in Adv. Stud. Pure Math. arXiv:1303.2404 [math.AG]

[F11] O. Fujino, Some remarks on the minimal model program for log canonical pairs, J. Math. Sci. Univ. Tokyo 22 (2015), no. 1, 149-192.

[F12] O. Fujino, Foundation of the minimal model program, preprint (2014), 2014/4/16, version 0.01 .

[F13] O. Fujino, On subadditivity of the logarithmic Kodaira dimension, preprint (2014). arXiv:1406.2759 [math.AG]

[F14] O. Fujino, On quasi-Albanese maps, preprint (2014). Kyoto-Math 2015-03

[F15] O. Fujino, Subadditivity of the logarithmic Kodaira dimension for morphisms of relative dimension one revisited, preprint (2015). Kyoto-Math 2015-02

[F16] O. Fujino, On semipositivity, injectivity and vanishing theorems, preprint (2015). arXiv:1503.06503 [math.AG] 
[FF] O. Fujino, T. Fujisawa, Variations of mixed Hodge structure and semipositivity theorems, Publ. Res. Inst. Math. Sci. 50 (2014), no. 4, 589-661.

[FFS] O. Fujino, T. Fujisawa, M. Saito, Some remarks on the semipositivity theorems, Publ. Res. Inst. Math. Sci. 50 (2014), no. 1, 85-112.

[FG] O. Fujino, Y. Gongyo, On images of weak Fano manifolds II, Algebraic and complex geometry, 201-207, Springer Proc. Math. Stat., 71, Springer, Cham, 2014.

[Fuk] S. Fukuda, A generalization of the Kawamata-Viehweg vanishing theorem after Reid, Comm. Algebra 24 (1996), no. 10, 3265-3268.

[HM] C. D. Hacon, J. M ${ }^{\mathrm{c}}$ Kernan, Boundedness of pluricanonical maps of varieties of general type, Invent. Math. 166 (2006), no. 1, 1-25.

[H1] R. Hartshorne, Residues and duality, Lecture notes of a seminar on the work of A. Grothendieck, given at Harvard 1963/64. With an appendix by P. Deligne. Lecture Notes in Mathematics, No. 20 Springer-Verlag, BerlinNew York 1966.

[H2] R. Hartshorne, Ample vector bundles, Inst. Hautes Études Sci. Publ. Math. No. 29 (1966), 63-94.

[HP1] A. Höring, T. Peternell, Minimal models for Kähler threefolds, to appear in Invent. Math. arXiv:1304.4013 [math.AG]

[HP2] A. Höring, T. Peternell, Mori fiber spaces for Kähler threefolds, J. Math. Sci. Univ. Tokyo 22 (2015), no. 1, 219-246.

[I1] S. Iitaka, Genus and classification of algebraic varieties. I. (Japanese), Sûgaku 24 (1972), no. 1, 14-27.

[I2] S. Iitaka, On logarithmic Kodaira dimension of algebraic varieties, Complex analysis and algebraic geometry, pp. 175-189, Iwanami Shoten, Tokyo, 1977.

[Ks] M. Kashiwara, A study of variation of mixed Hodge structure, Publ. Res. Inst. Math. Sci. 22 (1986), no. 5, 991-1024.

[Kw1] Y. Kawamata, Characterization of abelian varieties, Compositio Math. 43 (1981), no. 2, 253-276.

[Kw2] Y. Kawamata, Kodaira dimension of certain algebraic fiber spaces, J. Fac. Sci. Univ. Tokyo Sect. IA Math. 30 (1983), no. 1, 1-24.

[Kw3] Y. Kawamata, Semipositivity theorem for reducible algebraic fiber spaces, Pure Appl. Math. Q. 7 (2011), no. 4, Special Issue: In memory of Eckart Viehweg, 1427-1447.

[Kw4] Y. Kawamata, Hodge theory on generalized normal crossing varieties, Proc. Edinb. Math. Soc. (2) 57 (2014), no. 1, 175-189.

[Ko1] J. Kollár, Singularities of pairs, Algebraic geometry - Santa Cruz 1995, 221287, Proc. Sympos. Pure Math., 62, Part 1, Amer. Math. Soc., Providence, RI, 1997.

[Ko2] J. Kollár, Singularities of the minimal model program, Cambridge Tracts in Mathematics, 200. Cambridge University Press, Cambridge, 2013.

$[\mathrm{KM}]$ J. Kollár, S. Mori, Birational geometry of algebraic varieties, Cambridge Tracts in Mathematics, 134. Cambridge University Press, Cambridge, 1998.

[KP] S. Kovács, Z. Patakfalvi, Projectivity of the moduli space of stable log-varieties and subadditvity of log-Kodaira dimension, preprint (2015). arXiv:1503.02952 [math.AG]

[L] S. Lu, A refined Kodaira dimension and its canonical fibration, preprint (2002). arXiv:math/0211029 [math.AG] 
[Ma] K. Maehara, The weak 1-positivity of direct image sheaves, J. Reine Angew. Math. 364 (1986), 112-129.

[Mo] S. Mori, Classification of higher-dimensional varieties, Algebraic geometry, Bowdoin, 1985 (Brunswick, Maine, 1985), 269-331, Proc. Sympos. Pure Math., 46, Part 1, Amer. Math. Soc., Providence, RI, 1987.

[N1] N. Nakayama, The lower semicontinuity of the plurigenera of complex varieties, Algebraic geometry, Sendai, 1985, 551-590, Adv. Stud. Pure Math., 10, North-Holland, Amsterdam, 1987.

[N2] N. Nakayama, Zariski-decomposition and abundance, MSJ Memoirs, 14. Mathematical Society of Japan, Tokyo, 2004.

[PS] C. A. M. Peters, J. H. M. Steenbrink, Mixed Hodge structures, Ergebnisse der Mathematik und ihrer Grenzgebiete. 3. Folge. A Series of Modern Surveys in Mathematics, 52. Springer-Verlag, Berlin, 2008.

[RR] J. P. Ramis, G. Ruget, Complexe dualisant et théorèmes de dualité en géométrie analytique complexe, Inst. Hautes Études Sci. Publ. Math. No. 38 (1970), 77-91.

[RRV] J. P. Ramis, G. Ruget, J.-L. Verdier, Dualité relative en géométrie analytique complexe, Invent. Math. 13 (1971), 261-283.

[Schm] W. Schmid, Variation of Hodge structure: the singularities of the period mapping, Invent. Math. 22 (1973), 211-319.

[Schn] C. Schnell, Weak positivity via mixed Hodge modules, to appear in Hodge theory and Complex Algebraic Geometry (Columbus, 2013).

[St] J. H. M. Steenbrink, Mixed Hodge structure on the vanishing cohomology, Real and complex singularities (Proc. Ninth Nordic Summer School/NAVF Sympos. Math., Oslo, 1976), 525-563. Sijthoff and Noordhoff, Alphen aan den Rijn, 1977.

[SZ] J. H. M. Steenbrink, S. Zucker, Variation of mixed Hodge structure. I, Invent. Math. 80 (1985), no. 3, 489-542.

[Va] J. Varouchas, Sur l'image d'une variété kählérienne compacte, Fonctions de plusieurs variables complexes, V (Paris, 1979-1985), 245-259, Lecture Notes in Math., 1188, Springer, Berlin, 1986.

[Vd] J.-L. Verdier, Base change for twisted inverse image of coherent sheaves, 1969 Algebraic Geometry (Internat. Colloq., Tata Inst. Fund. Res., Bombay, 1968) pp. 393-408, Oxford Univ. Press, London.

[V1] E. Viehweg, Die Additivität der Kodaira Dimension für projektive Faserräume über Varietäten des allgemeinen Typs, J. Reine Angew. Math. 330 (1982), 132-142.

[V2] E. Viehweg, Weak positivity and the additivity of the Kodaira dimension for certain fibre spaces, Algebraic varieties and analytic varieties (Tokyo, 1981), 329-353, Adv. Stud. Pure Math., 1, North-Holland, Amsterdam, 1983.

[V3] E. Viehweg, Weak positivity and the additivity of the Kodaira dimension. II. The local Torelli map, Classification of algebraic and analytic manifolds (Katata, 1982), 567-589, Progr. Math., 39, Birkhäuser Boston, Boston, MA, 1983.

[V4] E. Viehweg, Quasi-projective moduli for polarized manifolds, Ergebnisse der Mathematik und ihrer Grenzgebiete (3), 30. Springer-Verlag, Berlin, 1995. 
Department of Mathematics, Graduate School of Science, Kyoto UNIVERSITY, KYOTO 606-8502, JAPAN

E-mail address: fujino@math.kyoto-u.ac.jp 